\title{
GLOBAL RESPONSE OF THE UPPER THERMOSPHERIC WINDS TO LARGE ION DRIFTS IN THE JOVIAN OVALS
}

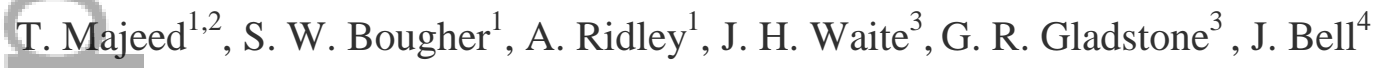 \\ $=$ \\ ${ }^{1}$ University of Michigan, 2455 Hayward St., Ann Arbor, Michigan, 48109-2143 USA \\ ${ }^{2}$ American University of Sharjah, P.O. Box 26666, Sharjah, UAE \\ ${ }^{3}$ Southwest Research Institute, 6220 Culebra Road, San Antonio, Texas 78238, USA \\ ${ }^{3}$ National Institute of Aerospace, 100 Exploration way, Hampton, VA 23666, USA

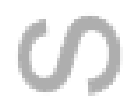

Short title: Neutral Winds in the Jovian Ovals

Correspondence to: T. Majeed at tmajeed@aus.edu or tariqm@umich.edu

Index Terms:

(1)

[1] Abstract: We use our fully coupled 3-D Jupiter Thermosphere General Circulation Model (JTGCM) to quantify processes which are responsible for generating neutral 1 winds in Jupiter's oval thermosphere from $20 \mu$ bar to $10^{-4}$ nbar self-consistently with the thermal structure and composition. The heat sources in the JTGCM that drive the global circulation of neutral flow are substantial Joule heating produced in the Jovian ovals by imposing high speed anticorotational ion drifts $\left(\sim 3.5 \mathrm{kms}^{-1}\right)$ and charged particle heating from auroral processes responsible for bright oval emissions. We find that the zonal flow of neutral winds in the auroral ovals of both hemispheres is

This is the author manuscript accepted for publication and has undergone full peer review but has not been through the copyediting, typesetting, pagination and proofreading process, which may lead to differences between this version and the Version of Record. Please cite this article as doi: 10.1002/2015JA021328

This article is protected by copyright. All rights reserved. 
primarily driven by competition between accelerations resulting from Coriolis forcing and ion drag processes near the ionospheric peak. However, above the ionospheric peak $(<0.01 \mu$ bar), the acceleration of neutral flow due to pressure gradients is found to be the most effective parameter impacting zonal winds, competing mainly with acceleration due to advection with minor contributions from curvature and Coriolis $=$ forces in the southern oval, while in the northern oval it competes alone with considerable Coriolis forcing. The meridional flow of neutral winds in both ovals in the JTGCM is determined by competition between meridional accelerations due to Coriolis forcing and pressure gradients. We find that meridional flow in the lower thermosphere, near the peak of the auroral ionosphere, is poleward, with peak wind speeds of $\sim 0.6 \mathrm{kms}^{-1}$ and $\sim 0.1 \mathrm{kms}^{-1}$ in the southern and northern oval, respectively. The corresponding subsiding flow of neutral motion is $\sim 5 \mathrm{~ms}^{-1}$ in the southern oval, while this flow is rising in the northern oval with reduced speed of $\sim 2 \mathrm{~ms}^{-1}$. We also find that the strength of meridional flow in both auroral ovals is gradually weakened and turned equatorward near $0.08 \mu$ bar with wind speeds up to $\sim 250 \mathrm{~ms}^{-1}$ (southern oval) and $\sim 75 \mathrm{~ms}^{-1}$ (northern oval). The corresponding neutral motion in this region is upward, with wind speeds up to $4 \mathrm{~ms}^{-1}$ in both ovals.

\section{Introduction}

[2] The Jovian observations of high-latitude regions have shown a strong electromagnetic coupling between Jupiter's magnetosphere and its ionosphere [Broadfoot 
et. al., 1979; Waite et al., 2000; Waite et al., 2001; Waite and Lummerzheim et al., 2002;

Gladstone et al., 2002; Stallard et al., 2001, 2002; Cravens et al., 2003; Gustin et al., 2004a,b, 2006; Elsner et al., 2005; Ajello et al., 2005; Pryor et al., 2005; Kharchenko et al., 2006; Grodent et al., 2008; Radioti et al., 2008; Gérard et al., 2013; Vogt et al., 2011; and references therein]. This coupling injects significant amounts of energy into the Jovian auroral atmosphere in the course of rotation enforcement and plasma processes that take place in the near corotating middle magnetosphere [Khurana, 2001; Hill, 2001; Cowley and Bunce, 2001; Hill and Vasyliunas, 2002; Cowley et al., 2003a,b; Nichols and Cowley, 2004; Millward et al., 2005; Nichols et al., 2009; Yates et al., 2012; 2014; and references therein]. The breakdown of this corotation in the magnetodisk at around 20-30 $\mathrm{R}_{\mathrm{J}}$ (Jupiter radii) produces field-aligned current, and closure of this current in the Jovian ionosphere equatorwards across the main ovals gives rise to a mechanism that transfers angular momentum from the polar atmosphere to the magnetosphere. This leads to the establishment of an anticorotational high-latitude ion drift around the ovals resulting from magnetic momentum coupling between the equatorial plasma sheet within the middle magnetosphere and the Jovian upper atmosphere. Charged particles with energies up to a few hundreds of $\mathrm{keV}$ are accelerated downward in this process and produce ultraviolet emissions [Gustin et al., 2004a, b; 2006] as well as ionization and heating of the ambient $\mathrm{H}_{2}$ atmosphere [Waite et al., 1983; Grodent et al., 2001]. Thus, auroral processes taking place within the main ovals are responsible for the thermospheric ionization, yielding a peak in the ionosphere between 1 and $0.1 \mu$ bar $(\sim 350-450 \mathrm{~km}$ above the 1 bar pressure 
level), and are likely the source of fast ion flows with wind speeds $>1 \mathrm{~km} \mathrm{~s}^{-1}$ in Jupiter's ionosphere.

[3] The middle magnetospheric plasma flows at Jupiter observed by Voyager and Galileo spacecraft [e.g., Eviatar and Barbosa, 1984; Kane et al., 1995; Krupp et al., 2001; Frank and Paterson, 2001] showed subcorotation of $E$ x $B$ drifting plasma,

yielding electric field convection to ionospheric heights, thereby producing ionospheric currents. The horizontal closure of these currents in the Jovian ovals creates frictional motion of the rotating ions and the background neutrals, causing Joule heating [see Cowley et al., 2008 for references]. The impact of high-latitude Joule and charged particle heating on the auroral thermosphere via the Coriolis force may produce meridional flow as a result of accelerating neutrals by ion drag and other processes. Achilleos et al. [1998] was the first to show how this flow effectively controls the global ionization and thermospheric heat budget on Jupiter. Millward et al. [2005] employed Achilleos et al.'s model and showed that ions with drift speeds of $\sim 1 \mathrm{~km} \mathrm{~s}^{-1}$ in and around the Jovian ovals can generate thermospheric neutral winds with speeds up to $1 \mathrm{~km} \mathrm{~s}^{-1}$. Later, Bougher et al. [2005] investigated that these winds near the auroral ionospheric peak may have great impact on the ion momentum forcing and the Joule heating.

[4] Current modeling of these thermospheric winds at Jupiter raises concerns whether these winds [Millward et al., 2005; Bougher et al., 2005; Majeed et al., 2005, 2009; Smith et al., 2007; Smith and Aylward, 2009, Tao et al., 2009; 2014; Yates et al., $2012,2014]$ really play a significant role in modifying the global heat budget or whether 
they only contribute locally to the elevated temperature observations in auroral and equatorial thermospheres, i.e., creating a mechanism that overheats the polar regions and enhances the equatorial cooling adiabatically. The fast Jovian rotation $\left(\sim 3 \mathrm{~km} \mathrm{~s}^{-1}\right)$ at the oval latitudes produces a strong Coriolis force. The ion drifts at these latitudes resulting from the magnetic coupling between the Jovian ionosphere and magnetodisk drags the neutral gas in the same direction as the ions, forming an electrojet near the height of the peak electron density. The Coriolis force acts on the electrojet and tends to deflect it toward the Jovian pole. If this deflection is overwhelmed by the meridional pressure gradients, it would produce equatorward winds necessary to transport high-latitude heat to the low latitude regions [Bougher et al., 2005; Majeed et al., 2005, 2009; Tao et al., 2009; 2014]. Conversely, if Coriolis forcing remains strong in the Jovian oval compared to that of the meridional pressure gradients, then the neutral winds would be blowing poleward of the main oval. Smith and Aylward [2009] and mostly recently Yates et al. $[2014 ; 2014]$ have shown that the anticorotational zonal winds generated by ion drag in their coupled ionosphere-thermosphere models are influenced by strong Coriolis forces and produce poleward flow throughout the Jovian oval.

[5] Recently, Tao et al. [2009] and Smith and Aylward [2009] presented their coupled magnetosphere-ionosphere-thermosphere models for the Jovian system. They obtained numerical solutions of the neutral dynamics and coupling current between the magnetosphere and ionosphere self-consistently with the thermospheric temperature and ionospheric conductance. The basic structure of these models is based on the middle 
magnetosphere model of Nichols and Cowley [2004] with an axisymmetric model of the entire magnetosphere developed by Cowley et al. [2005] and the Saturn model of Smith and Aylward [2008] with minimal input from the global circulation model of Achilleos et al. [1998]. The auroral inputs of diffuse auroral conditions used by Smith and Aylward (2009), representing an unstructured aurora in the Jovian polar cap, with a total characteristic energy of $18.1 \mathrm{keV}$ of precipitating particles and a corresponding energy flux of $30.5 \mathrm{erg} \mathrm{cm}^{-2} \mathrm{~s}^{-1}\left(1 \mathrm{erg} \mathrm{cm}^{-2} \mathrm{~s}^{-1}=1 \mathrm{~mW} \mathrm{~m}^{-2}\right)$ were taken from the 1-D electron transport model of Grodent et al. [2001] and scaled to the global pattern of auroral conductance. Tao et al., [2009] also assumed diffuse auroral conditions for their model with precipitated electron energy of $8.7 \mathrm{keV}$, while the corresponding electron flux was calculated as a function of field-aligned current following a suggestion from Nichols and Cowley 2004]. Their model also has a capability of calculating radial currents at large distances in the Jovian equatorial plasma disk with varying initial energy spectrum of precipitating electrons up to $200 \mathrm{keV}$.

[6] The coupled models described the transfer of angular momentum radially in the magnetosphere and meridionally in the thermosphere in a system in which the magnetospheric flow of plasma is in corotation with the thermospheric wind system. This self-consistent rotational coupling between the two rotating systems has great implications to the neutral dynamics of the Jovian thermosphere through ion drag and Joule heating; i.e., the plasma flow through the field-aligned current in the auroral ionosphere that mapped to the magnetosphere, and the radial current in different regions 
of the magnetosphere [cf., Ray et al., 2014]. The thermospheric response to this coupling shows that while westward flow induced by ion drag is acted on by Coriolis forces and generates strong poleward winds throughout the polar region coupled to sub-corotating ions [Bougher et al., 2005; Yates et al., 2012; 2014], a divergence in this poleward flow occurs in the cororating oval region yielding an upwelling of neutrals from altitudes of maximum electron density [Bougher et al., 2005; Majeed et al., 2009; Tao et. al., 2014]. This upwelling expands neutral gas in a region just equatorward of the main ovals, cooling the region adiabatically [Majeed et al., 2005; Achilleos et al., 1998]. Clearly, dynamics of the inner and middle magnetosphere of Jupiter is critical in driving the global thermal structure, neutral wind velocity, and ionospheric conductance in the Jovian thermosphere.

[7] Tao et al. [2009] calculated the neutral temperature of about $1020 \mathrm{~K}$ and 750

$\mathrm{K}$ in the polar region of Jupiter's northern hemisphere and near the Jovian equator, respectively, in a pole-to-equator circulation of neutral flow generated by the action of Coriolis forces on the equatorward-directed meridional winds. In order to obtain these temperatures, they have used an alternative source of heating in the upper thermosphere by sonic waves originating from the Jovian lighting. The neutral flow in this process tends to reduce the rotation speed of both the Jovian thermosphere and middle magnetosphere (radial distance <30 RJ), giving rise to energy partitioning of the coupled system. Tao et al. [2009] found that the mechanical energy generated in the coupled magnetosphere-ionosphere system is used to accelerate magnetospheric plasma into the 
Jovian thermosphere equatorward of $74^{\circ} \mathrm{N}$ latitude. However, above this latitude, the energy is consumed in the polar thermosphere via Joule heating or ion drag. Thus the neutral wind system in the Jovian thermosphere plays a critical role in determining the consumption of global energy in the process of accelerating neutral flow. Tao et al. [2009] simulated an anticorotational zonal neutral wind speed of $\sim 742 \mathrm{~m} \mathrm{~s}^{-1}$ in the Jovian " upper atmosphere at $\sim 74^{\circ} \mathrm{N}$ latitude and a reduced speed of $\sim 100 \mathrm{~m} \mathrm{~s}^{-1}$ near equator. At the same time their model yielded an equatorward-directed meridional wind speed of $\sim 72$ $\mathrm{m} \mathrm{s}^{-1}$ near $74^{\circ} \mathrm{N}$ latitude and a poleward-directed meridional wind speed of $\sim 17 \mathrm{~m} \mathrm{~s}^{-1}$, in addition to polar upwelling of $\sim 0.75 \mathrm{~m} \mathrm{~s}^{-1}$ and subsequent convergence and downwelling of about $0.4-0.2 \mathrm{~m} \mathrm{~s}^{-1}$ near the Jovian mid-latitude and equatorial regions. The thermospheric temperatures and neutral wind speeds simulated by Tao et al. [2009] are roughly in agreement with those simulated by Bougher et al. [2005] and Majeed et al. [2005, 2009]. The simulated temperatures by Tao et al. [2009] are also largely within the observational limits derived from multi-spectral observations of the Jovian aurora [Miller et al., 1997; Stallard et al., 2001, 2002; Gustin et al., 2004a,b; Raynaud et al., 2003, 2004] and in-situ observation of neutral temperatures near the Jovian equator [Sieff et al., 1998].

[8] In order to simulate similar temperatures, Smith and Aylward [2009] claimed that their model requires an additional Joule heating induced presumably by a component of rapidly fluctuating electric fields to activate the Jovian wind system both in the polar region and near equator for transporting heat to the cooler regions by meridional 
advection. To obtain an agreement with temperatures derived from spectroscopic observations of $\mathrm{H}_{3}{ }^{+}$aurora [Lam et al., 1997; Stallard et al., 2002], they imposed fluctuating electric fields at latitudes $>74^{\circ} \mathrm{N}$, linking the Jovian polar cap regions to the outer magnetosphere at radial distances $>80 \mathrm{R}_{\mathrm{J}}$. One of the consequences of this approach is that it drives a broader and less intense peak of the field-aligned current at greater radial distances in the Jovian magnetosphere. This may broaden and weaken the main auroral oval in addition to moving its location slightly poleward, which is in contradiction with observations [Grodent et al., 2003; Gérard et al., 2003; Vogt et al., 2011]. To fit the equatorial temperature profile observed with the Galileo probe, Smith and Aylward [2009] applied electric field fluctuations equatorward of $70^{\circ} \mathrm{N}$ latitude with extra heat that seems to be greatly dispersed across the Jovian equator by the thermospheric winds. According to Smith and Aylward [2009], this extra heat can also cause poleward blowing winds in the model that may result in super-corotation of the polar latitudes coupled with the inner magnetosphere, in contradiction to the plasma observations of Jupiter's dayside magnetosphere [McNutt et al., 1979]. Clearly, the magnetosphere-ionosphere-thermosphere models presented by Tao et al. [2009] and Smith and Aylward [2009] are in early stages of development similar to the Jupiter Thermosphere General Circulation Model (JTGCM) [Bougher et al. 2005; Majeed et al. 2005, 2009]. Much of the numerical framework of these models has been adapted from terrestrial GCMs, using simplified versions of several components and parameterizations 
of several quantities in addition to several assumptions which may not be realistic in absence of observational information.

[9] Most recently, Tao et al. [2014] added a time-varying component of the solar EUV flux described by variations in the $\mathrm{F}_{10.7}$ index in their 2009 model to investigate short-term and long-term variations in the Jovian thermospheric temperatures and neutral wind speeds. They found that the Jovian ionospheric conductance is increased for large values of the solar EUV fluxes $\left(\mathrm{F}_{10.7}>160\right)$ at Jupiter. The increased conductance is used to intensify the acceleration of magnetodisk plasma and generate large field-aligned currents at ionospheric heights. This gives rise to faster neutral wind speeds near the Jovian mid-latitudes immediately in response to increasing the solar EUV fluxes. Another consequence of increased ionospheric conductance is that it enhances the net heat induced by auroral particle impact and Joule heating, warming the Jovian polar region and developing large meridional pressure gradients to efficiently propagate highlatitude heat towards the Jovian equator.

[10] In a parallel work Yates et al. [2014; see also Yates et al. 2012], using Smith and Aylward [2009] model, studied the Jovian neutral dynamics and thermal structure in response to magnetospheric compression and expansion caused by transient variations in the solar wind dynamic pressure. They found that during an event of transient compression, an increase in corotation speed between the Jovian ionosphere and middle magnetospheric plasma could yield a strong coupling current and provide a substantial increase in auroral precipitation with electron energies approaching several hundreds of 
$\mathrm{keV}$ into Jupiter's main auroral oval whose brightness could exceed $2000 \mathrm{kR}$. Yates et al. [2014] also calculated meridional speeds of the neutral flow ranging from $100-200 \mathrm{~ms}^{-1}$, which are large enough to effectively transport auroral energy across the planet, but not as sufficient to account for the high temperatures observed near the Jovian equator in agreement with Smith et al. [2007]. In contrast to this, Yates et al. [2014] found that the $=$ degree of plasma corotation decreases as the Jovian magnetosphere expands gradually, altering the neutral dynamics and energy balance of the planetary system. The ionneutral coupling during this expansion event modestly increases the combined particle heating, Joule heating and ion drag energy dissipation in the Jovian high-latitude region, causing the local forces to act efficiently in accelerating the neutral flow meridionally to polar cap region. Yates et al. [2014] also showed that the main auroral oval emissions become less intense ( $25 \%$ of the compressional event) in response to reduced coupling current. Since the current version of the JTGCM does not include either time-variation of the solar EUV fluxes or the component that invokes the solar wind dynamic pressure in the model, it is not possible for us to directly compare our results with those obtained by Tao et al. [2014] and Yates et al. [2014]. However, the earlier versions of the models [Tao et al., 2009; Smith and Aylward, 2009] are relevant to the JTGCM and we will compare the results of this investigation with the results of these models where necessary. [11] There are major differences between the JTGCM [Bougher et al., 2005] and Smith's and Tao's models. One of them is the use of axial symmetry or mirror symmetry assumption regarding the Jovian thermosphere and magnetosphere with a spin-aligned 
dipole magnetic field whose equatorial strength is assumed to be $\mathrm{B}_{\mathrm{j} 0}=4.35 \times 10^{-4} \mathrm{~T}$ and a constant field of $2 \mathrm{~B}_{\mathrm{jo}}$ at high-latitude polar ionosphere. In order to map rotation velocities along the field lines between the equatorial magnetosphere and the highlatitude ionosphere, both Smith and Aylward and Tao et al., adapted a flux function from the work of Nichols and Cowley [2004] defined separately for the Jovian ionosphere and magnetosphere. To obtain a coupled ionosphere-magnetosphere system, they made these functions equal to each other in an attempt to map radial distance in the Jovian magnetospheric equatorial plane to the magnetically conjugate ionospheric colatitude. According to Hill [2001], at radial distances $>20 \mathrm{R}_{\mathrm{J}}$ where the magnetic field is considerably low and the plasma speed in the magnetodisk required to maintain corotation with the neutral atmosphere is too large, the plasma lags behind the Jovian field lines magnetically connected to oval locations and generates field-aligned current. The upward field-aligned current in the magnetodisk is responsible for the downward precipitation of energetic electrons in the auroral ovals through field lines that cross the Jovian magnetic equator. The closing of this current across the high-latitude ionosphere for the radial distances beyond $30 \mathrm{R}_{\mathrm{J}}$ enforces the Jovian ions to drift counter to the planetary direction under the combined influence of the Jovian magnetic field and the ionospheric electric field. The drifting ions drag the neutrals in the same anticorotational direction via ion-neutral collisions. This frictional motion between ions and neutrals causes resistive heating or Joule heating. Joule heating and ion drag in Smith 
and Aylward and Tao et al. models are calculated by using the plasma rotation velocity combined with ionospheric conductance and thermospheric wind speeds.

[12] On the other hand, in the JTGCM, a convection electric potential is calculated using a simple ionospheric-magnetospheric model based on Voyager measurements of ion convection in the outer magnetosphere [cf. Eviatar and Barbosa,

1984]. Subsequently, the VIP4 magnetic field model [Connerney et al., 1998] is used to map this magnetospheric electric potential pattern to ionospheric altitudes at auroral oval latitudes (see Appendix A2 for details). The parameterization of ion-drag and Joule heating in the JTGCM code is based on the formulation described by Roble and Ridley [1987] for the $10^{\circ}$ offset Jovigraphic and Jovimagnetic poles (see Appendix A1 for details). This gives rise to underlying asymmetries in Joule heating and ion drag processes owing to hemispheric differences in the VIP4 magnetic field which is missing in the models developed by Smith and Aylward [2009] and Tao et al. [2009].

[13] Another major difference between the JTGCM and Smith and Aylward, and Tao et al., models is the parameterization of auroral heating caused by energetic particle precipitation in the Jovian high-latitude ionosphere. JTGCM uses the discrete auroral representation of the main auroral oval with precipitating particle energy and corresponding energy flux much larger than those used in Smith and Aylward [2009] and Tao et al., [2009] (see Table 1). It is important to note that Tao et al. [2009] have used an alternative source of heating in the upper thermosphere by sonic waves originating from Jupiter's lighting in an attempt to explain the measured temperatures. However, these 
authors have neglected the zonal geopotential gradient terms in their model while solving the energy transport and momentum forcing equations. The resulting impact of neglecting this important term on the distribution of the Jovian thermospheric temperature and neutral wind speeds is quite severe especially at auroral latitudes where large temperature gradients are developed.

$=$

[14] The three models mentioned above also calculated field-aligned current and Pedersen conductivity in the Jovian auroral oval. Smith and Aylward [2009] using their comprehensive ionosphere-magnetosphere-thermosphere model that incorporates an extra source of Joule heating due to electric field fluctuations in both the auroral and equatorial regions have shown that the peak values of field-aligned current and Pedersen conductivity are in the range $0.25-0.28 \mu \mathrm{A} \mathrm{m}^{-2}$ and $1.3-1.5$ mho, respectively. These values are in good agreement with those calculated by Nichols and Cowley [2004] but larger by a factor of $\sim 4-5$ larger than those obtained by Tao et al. [2009]. JTGCM also estimated field-aligned current of $\sim 4.4 \mu \mathrm{A} \mathrm{m} \mathrm{m}^{-2}$ and Pedersen conductivity of $\sim 12$ mho [Bougher et al., 2005; Majeed et al., 2009], using auroral conditions of intense particle precipitation [Grodent et al. 2001] and an estimated mapping of the Jovian magnetospheric ion convection at auroral latitudes discussed above. These estimated values of the JTGCM field-aligned current and Pedersen conductivity are approximately a factor of 15 to 8 larger than those simulated by Smith and Aylward [2009] and even larger than those obtained by Tao et al. [2009]. Table 1 summarizes the difference between JTGCM and these two models based on various factors including, the difference 
in auroral energy inputs, the use of different magnetic field models, the difference in plasma drift velocities, and corresponding Joule heating rates.

[15] As noted above, substantially large ion drag and Joule heating in the JTGCM are expected as a result of the large auroral conductivity and convection velocity at ionospheric heights derived from Voyager measurements of the bulk plasma velocity vectors in the inner and outer magnetosphere of Jupiter. It is worth noting that the plasma flow processes within the Jovian magnetosphere and auroral processes in Jupiter's thermosphere are poorly understood owing to limited knowledge of the global magnetic field configuration of the Jovian system. Thus the estimated ion drifts and the energy spectrum of the precipitating charged particles employed in the JTGCM are the most ambiguous input parameters. Gustin et al. [2004b] found that the bright FUV Jovian emissions in the main oval are excited by the auroral processes of charged particles with energies up to $200 \mathrm{keV}$ and associated energy fluxes up to $200 \mathrm{ergs} \mathrm{cm}^{-2} \mathrm{~s}^{-}$ - While local time asymmetries in oval emissions with less energy fluxes have been observed [Gustin et al., 2006; Radioti et al., 2008; Nichols et al., 2009] perhaps in response to corresponding asymmetries in the Jovian magnetosphere [Ray et al., 2014], what causes these local time asymmetries is still under debate. Moreover, CFHT infrared observations of the Jovian auroral hot spots suggest a thermosphere with temperatures in excess of $1300 \mathrm{~K}$ [Raynaud et al., 2004b], indicating strong ion drag forcing and Joule heating that is likely due both to auroral particle precipitation and high-latitude ion drifts [Bougher et al. 2009; Majeed et al., 2009]. A strong north-south asymmetry in the polar 
region temperatures, due to hemispheric differences in VIP4 magnetic field topologies and the Pedersen conductivities of the northern and southern oval regions, is also predicted [Bougher et al., 2009].

[16] The assumed energy spectrum listed in Table 1 is based on the 1-D electron transport calculation of Grodent et al. [2001], which has been used to interpret auroral $=$ temperature observations, and is within the range described above. This auroral input, combined with the ion drift pattern (see Appendix A), is responsible for generating the large ionospheric conductivity and strong ion drag and Joule heating within the main Jovian ovals. Our JTGCM simulation uses these large inputs to provide an extreme case study for understanding the neutral momentum forcing processes at auroral latitudes near the Jovian ionospheric peak and their impact on the neutral dynamics at low and midlatitudes. The extreme values of input parameters incorporated in this simulation as listed in Table 1 compared to other models are likely an upper limit to what is possible. A downward scaling of these parameters has been adopted to explain temperature observations in Jupiter's thermosphere [Bougher et al., 2005; Majeed et al., 2005, 2009], which is in comparable with early studies of terrestrial thermosphere with general circulation models [Dickinson et al., 1981; Roble et al., 1988]. The input parameters used in the JTGCM and other models [Smith et al., 2009; Tao et al., 2009, 2014; Yates et $a l ., 2012 ; 2014]$ will be reevaluated after the Juno spacecraft, with its unique orbit, has explored Jupiter's polar magnetosphere. 


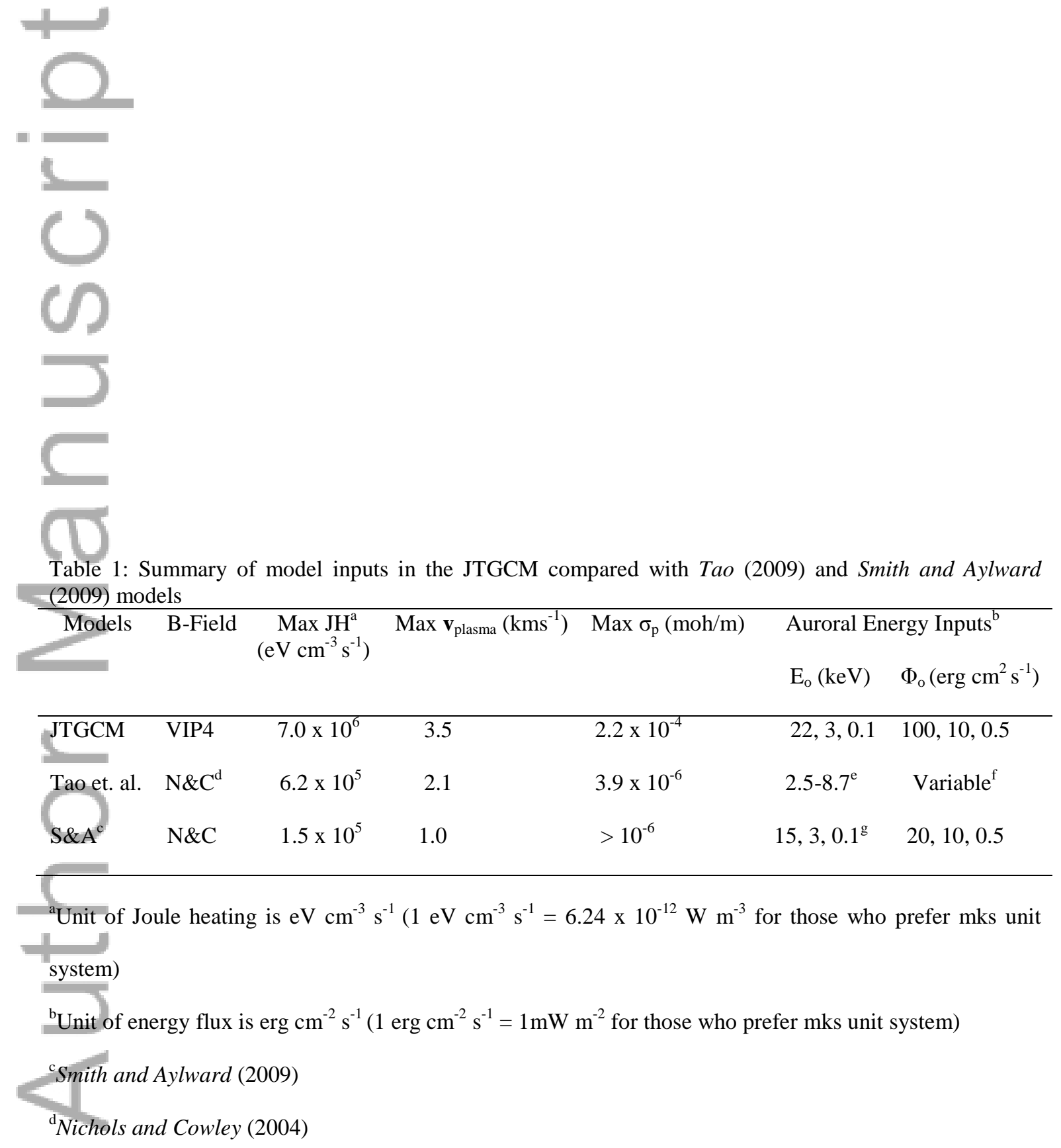


eaccording to the equation 7 of Tao et al. (2009) for variable field-aligned current

${ }^{f}$ as a function of field-aligned current

${ }^{g}$ combined Maxwellian and kappa energy distribution

\section{Jupiter Thermospheric Wind Measurements}

= [17] Theoretical and observational studies have been performed to obtain information on aurorally-driven thermospheric winds at Jupiter [Sommeria et al., 1995; Achilleos et al., 1998; Rego et al., 1999; Stallard et al., 2001, 2002, 2003; Millward et al., 2005;

Chaufray et al., 2010, 2011]. The modeling of large aurora-induced dynamics on Jupiter has led observers to provide necessary tools for measuring these winds using highresolution spectrometry. UV and IR techniques are employed to observe high-resolution images of auroral ovals. The analysis of these images provides information on the magnitudes of the Jovian wind speeds. The high-altitude neutral wind speeds between 4 and $8 \mathrm{kms}^{-1}$ have been obtained from the modeling of asymmetric Ly- $\alpha$ auroral line profiles [e.g., Prangé et al., 1997; Gladstone et al., 1998; Chaufray et al., 2010]. The direct ion and neutral wind speeds can be obtained from the measured Doppler shifts of strong $\mathrm{H}_{3}{ }^{+}$and $\mathrm{H}_{2}$ emission lines. The first evidence of these wind speeds in the Jovian northern auroral oval was presented by Rego et al. [1999]. They analyzed high-resolution $-\mathrm{H}_{3}{ }^{+}$emission lines near $3.9 \mu \mathrm{m}$ observed with CSHELL on the InfraRed Telescope Facility (IRTF) and derived an anticorotational ion wind of magnitude more than $2 \mathrm{kms}^{-1}$ in the main oval ionosphere. With the same instruments on IRTF, Stallard et al. [2001, 
2002, 2003] detected auroral images of Jupiter's northern hemisphere during an auroral event which lasted for more than three days. The analysis of these IR images yielded $\mathrm{H}_{3}{ }^{+}$ wind speeds of $\sim 0.5 \mathrm{kms}^{-1}$ and $\sim 1 \mathrm{kms}^{-1}$ at the beginning and at the end of the event, respectively.

[18] Recently, in parallel with the work of Rego et al., and Stallard et al., Chaufray et al. [2011] analyzed infrared observations of the Jovian aurora obtained with the Fourier Transform Spectrometer (FTS/BEAR) instrument at the Canada-France-Hawaii Telescope (CFHT) to derive the neutral and ion wind velocities in the northern auroral region of Jupiter. In BEAR mode, FTS obtained interferograms for each pixel of a $256 \mathrm{x}$ 256 element with spectral resolution of $0.2 \mathrm{~cm}^{-1}$, corresponding to a resolving power of 25,000. This allows Doppler shifts of $\mathrm{H}_{3}{ }^{+}$and $\mathrm{H}_{2}$ lines near $2 \mu \mathrm{m}$ to be determined to an accuracy of $\sim 0.4 \mathrm{~km} \mathrm{~s}^{-1}$ by centroiding. This technique provides an excellent opportunity

to study the dynamics of the Jovian upper atmosphere over the 24 ''-diameter field of view.

[19] Chaufray et al. [2011] used narrow-band filter F2.12 and recorded simultaneous observations of emission lines from $\mathrm{H}_{2} \mathrm{~S}_{1}(1)$ quadrupole and $\mathrm{H}_{3}{ }^{+} 2 \mathrm{v}_{2} \mathrm{R}(7,7)$ band on October 10, 2000 at $13 \mathrm{~h} 16$ (observation \#8) and 14h32 (observation \# 9). The total acquisition time during one observation was about 60 minutes, corresponding to a longitudinal smearing of approximately $36^{\circ}$. The Gaussian centroiding was used to derive velocities from Doppler shifts that were just a fraction of the instrumental resolution. Because of planetary rotation that introduces a smearing of $36^{\circ}$ longitude in 
the observed atmospheric structures, Chaufray et al. [2011] carefully adjusted systematic uncertainty resulting from paraboloid correction and inferred wind velocities for each observation averaged over the northern high-latitude region confined to $\sim 65-75^{\circ}$ latitudes and $\sim 140-180^{\circ}$ longitudes. An anticorotational $\mathrm{H}_{3}{ }^{+}$wind velocity from observation \#8 was observed, with an average magnitude of $3.1 \pm 0.4 \mathrm{kms}^{-1}$ approximately $1 \mathrm{kms}^{-1}$ and 2 $=\mathrm{kms}^{-1}$ larger than those derived by Rego et al. [1999] and Stallard et al. [2003], respectively. The corresponding magnitude of the average $\mathrm{H}_{2}$ wind velocity from this observation is derived to be only $0.6 \pm 0.4 \mathrm{kms}^{-1}$. For observation \#9, which was taken about one hour after the observation \#8, Chaufray et al. [2011] found that the $\mathrm{H}_{3}{ }^{+}$and $\mathrm{H}_{2}$ wind speeds were reduced close to instrumental uncertainties, indicating that the winds in the Jovian aurora had varied with time as previous observations suggest [Rego et al., 1999; Stallard et al., 2003].

[20] The difference between the magnitudes of the ion and neutral wind velocities may be interpreted as being due to the difference in emission altitudes [Grodent et al., 2001; Bougher et. al., 2005]. However, most recently Uno et al., [2014] based on their analysis of the observed $\mathrm{H}_{3}{ }^{+}$and $\mathrm{H}_{2}$ infrared aurorae of the northern polar region have shown that the source of the peak emission of both $\mathrm{H}_{3}{ }^{+}$and $\mathrm{H}_{2}$ can be located near the same altitudes, even though their observed auroral morphology in the Jovian polar region is very different. The observed $\mathrm{H}_{3}{ }^{+}$band emissions are mostly from the polar cap region consistent with observations of Satoh and Connerney [1999] while the $\mathrm{H}_{2}$ line emission is confined to latitudes within and poleward of the main auroral oval. Uno et al., [2014] 
have reproduced the observed emissivity profile of $\mathrm{H}_{3}{ }^{+}$with an emissivity model developed by Tao et al. [2011; 2012] and determined that the average altitude of the peak emission of $\mathrm{H}_{3}{ }^{+}$overtone and hot overtone lines is $\sim 580 \pm 160 \mathrm{~km}$ which is statistically similar to that of $\sim 640 \pm 90 \mathrm{~km}$ for the $\mathrm{H}_{2} \mathrm{~S}_{1}$ lines [e.g., Cravens, 1987; Kim et al., 1988].

\section{A Brief Model Description}

[21] The major characteristics of the JTGCM code and its objectives have been described in detail by Bougher et al. [2005]. Here, we briefly review important features of the JTGCM that are relevant to this study. The JTGCM is a fully-coupled 3D model which contains 39 vertical pressure levels with each level separated by 0.5 pressure scale heights on a $5^{\circ}$-latitude by $5^{\circ}$-longitude grid. It simulates the global distributions of upper atmospheric densities and neutral temperatures self-consistently with horizontal neutral winds in addition to vertical winds. The differential equations to be solved to

derive these simulations include: the thermodynamic equation, zonal and meridional momentum equations, the coupled continuity-diffusion equations, the hydrostatic equation, and the continuity equation (for vertical velocity) [cf., Dickinson et al., 1981; Roble et al., 1988]. The equations of interest for this paper are zonal and meridional momentum equations. The numerical solutions of these differential equations describe neutral flow of the general circulation in the east-west and north-south directions, respectively. The vertical velocity calculated in the JTGCM is characterized as upwelling or downwelling of the neutral atmospheric motion, giving rise to atmospheric 
expansion or compression. The governing equations for momentum forcing are described as follows:

\section{Zonal (eastward) momentum equation}

$=$

1

$$
\left.\frac{\partial u}{\partial t}=\frac{g e^{z p}}{p_{o}} \frac{\partial}{\partial z_{p}}\left(\frac{K_{M}}{H} \frac{\partial u}{\partial z_{p}}\right)+\frac{u v}{r} \tan \phi+f v-\left[V . \nabla u+w \frac{\partial u}{\partial z_{p}}\right]-\frac{1}{r \cos \phi} \frac{\partial \Phi}{\partial \lambda}+\left[\left(v_{i}-v\right) \alpha_{x y}+\left(u_{i}-u\right) \alpha_{x x}\right)\right]
$$

\section{Meridional (northward) momentum equation}

$$
\frac{\partial v}{\partial t}=\frac{g e^{z p}}{p_{o}} \frac{\partial}{\partial z_{p}}\left(\frac{K_{M}}{H} \frac{\partial}{\partial z_{p}}\right)-\frac{u^{2}}{r} \tan \phi-f u-\left[V . \nabla v+w \frac{\partial v}{\partial z_{p}}\right]-\frac{1}{r} \frac{\partial \Phi}{\partial \phi}+\left[\left(v_{i}-v\right) \alpha_{y y}-\left(u_{i}-u\right) \alpha_{y x}\right]
$$

Where $z_{p}$ is $\log$ pressure $\left(z_{p}=\ln \left(p_{o} / p\right), p_{o}\right.$ is reference pressure $\left(4.5 \mu\right.$ bar, where $\left.z_{p}=0\right), \phi$ is latitude, $\lambda$ is longitude, $\Phi$ is geopotential, $f$ is Coriolis parameter, $r$ is radial distance to the center of the planet, $u$ is zonal velocity, $v$ is meridional velocity, $w$ is vertical velocity $\left(d z_{p} / d t\right)$ (vertical motion), $g$ is acceleration of gravity, $K_{M}$ is molecular viscosity, $H$ is mean pressure scale height, V. $\nabla=u / r \cos \phi \partial / \partial \lambda+v / r \partial / \partial \phi, \alpha_{x x}, \alpha_{x y}, \alpha_{y y}$ and $\alpha_{y x}$ are iondrag parameters (tensors). The zonal $\left(\mathrm{u}_{\mathrm{i}}\right)$ and meridional $\left(\mathrm{v}_{\mathrm{i}}\right)$ components of the ion drift velocity are described in appendix A1. The first through sixth terms of the right-hand 
side of above momentum forcing equations are described as viscosity, curvature, Coriolis, hydrodynamic advection, geopotential gradient, and ion-drag terms, respectively. The differential equations mentioned above are solved on the latitudelongitude grid with log-pressure coordinates, $z_{p}$, with each $z_{p}$ interval being one scale height at the local temperature.

$=$

[22] The lower boundary in the JTGCM is located at $20 \mu$ bar to include hydrocarbon cooling resulting from $\mathrm{C}_{2} \mathrm{H}_{2}$ and $\mathrm{CH}_{4}$ emissions at $12.6 \mu \mathrm{m}$ and $7.8 \mu \mathrm{m}$, respectively [cf., Drossart et al., 1993]. At the lower boundary the zonal, meridional, and vertical winds are assumed to be zero. The lower boundary conditions for the neutral temperature and neutral densities are based on Galileo probe results [Seiff et al., 1998] and Voyager data [Festou et al., 1981]. The upper boundary of the JTGCM is located at about $1.1 \times 10^{-4}$ nbar, which is high enough to take into account the heating produced by auroral particle precipitation [Grodent et al., 2001] and corresponding cooling resulting from near-IR emission from $\mathrm{H}_{3}{ }^{+}$bands [Drossart et al., 1989]. Vertical gradients in temperature and in the three-component neutral winds are set to zero at the upper boundary. The highlatitude heating rates are calculated with the 1-D auroral transport model of Grodent et al. [2001] to represent the discrete auroral conditions. These rates are parameterized in the JTGCM [cf., Bougher et al., 2005] and distributed symmetrically in $\lambda_{\text {III }}$ longitude along the main auroral ovals in the two hemispheres. The oval positions prescribed in the JTGCM are based on the auroral morphology derived from the auroral images observed with UV imagers on board Hubble Space Telescope (HST) [Grodent et al., 2003]. For 
further details of the JTGCM boundary conditions and parameterizations of the Jovian ionosphere, and thermospheric heating and cooling rates the reader is referred to Bougher et al. [2005].

[23] Our aim in this paper is to discuss and quantify processes responsible for generating zonal and meridional components of the horizontal (neutral) wind velocity in the JTGCM simulation, which has been used to reasonably fit the thermospheric temperatures derived from multispectral observations of Jupiter's polar and equatorial regions. These fits were obtained by downward scaling of ion drifts and the total Joule heating produced in the Jovian ovals [Bougher et al., 2005; Majeed et al., 2009; 2005]. In this study a comparative analysis is made of the vertical profile of each acceleration term of the momentum equations, by using a full mapping of convection electric field which gives rise to large ion drifts (see Append A1), to show which processes are important in generating zonal and meridional neutral winds in each hemisphere's oval. The magnitudes of the vertical profiles of average neutral wind velocity for both the northern and southern ovals are obtained. The neutral wind speed for the northern highlatitude region mapped by the infrared observations taken with CFHT is also simulated in the JTGCM to explain the measured $\mathrm{H}_{2}$ wind speed.

\section{JT̈GCM Wind Simulations}

[24] In Figure 1 we show longitudinally averaged contours of neutral zonal, meridional, and vertical wind speeds in the Jovian thermosphere from $20 \mu$ bar to $1 \times 10^{-4}$ 
nbar. This view of latitudinal distribution describes how the thermospheric winds may vary in the JTGCM simulation which is driven by Joule heating and charged particle heating resulting from auroral precipitation in the main ovals. The fractional Joule heating has been shown to be an effective method in explaining the temperature observations at Jupiter's auroral and equatorial regions [Bougher et al., 2005; Majeed et al., 2005, 2009; Smith and Aylward, 2009; Tao et al., 2009]. The resulting global flow from this JTGCM simulation can be used to explain the observed north-south asymmetry in the $\mathrm{H}_{3}{ }^{+}$temperature distribution obtained by Miller et al. [1997] from spectral imaging of the Jovian atmosphere.

[25] In Figure 1a, we show the development of a very strong westward flowing zonal jet close to $70^{\circ} \mathrm{S}$ latitude at around $0.08 \mu$ bar $\left(Z_{p} \sim 4\right)$ level. This jet is primarily driven by the process of ion drag and corresponding Joule heating produced in the southern oval. Note that as the simulation progresses large meridional temperature gradients are created in the southern hemisphere in addition to a Coriolis torque that pushes the initially equatorward-directed flow toward the west [Bougher et al., 2005; Majeed et al., 2005, 2009]. The resulting westward flowing jet then spreads and largely dominates the zonal flow throughout the southern hemisphere for pressure $<0.05 \mu$ bar $\left(Z_{p}\right.$ $\geq 4.5$ ) with retrograde wind speeds, approaching more than $1.2 \mathrm{kms}^{-1}$ near the top of the atmosphere. This zonal flow in the JTGCM is accompanied by large equatorward meridional wind speeds in the range $300-400 \mathrm{~ms}^{-1}$ and upward wind speeds up to $5 \mathrm{~ms}^{-1}$ as shown in Figure 1b, and 1c, respectively. This type of neutral flow in the Jovian 
thermosphere has been shown to be responsible for transporting auroral heat towards the low and mid-latitudes [Majeed et al., 2005; 2009; Tao et al., 2009]. The westward flowing zonal jet in the lower thermosphere weakens to $\sim 100 \mathrm{~ms}^{-1}$ near $1 \mu$ bar $\left(Z_{p}=1.5\right)$ in the southern auroral region between $60^{\circ} \mathrm{S}$ and $80^{\circ} \mathrm{S}$, and the corresponding meridional flow turns poleward (Figure 1b), accompanied by a strong subsiding (Figure 1c) flow that results in poleward advection of heat for pressure $>0.08 \mu$ bar $\left(Z_{p}<4\right)$ [Majeed et al., 2009]. The dynamical heat resulting from this unique circulation of neutral flow is a direct consequence of ion drag forcing poleward of the southern oval. The neutral horizontal wind speeds (zonal plus meridional) up to $1.4 \mathrm{kms}^{-1}$ are simulated in the southern oval. These wind speeds are almost a factor of two faster than those simulated with the same JTGCM code with auroral forcing alone [Bougher et al., 2005], that is by neglecting the impact of Joule heating and ion drag on the thermospheric flow.

[26] A similar, though weaker, westward jet is formed in the northern hemisphere at around $60^{\circ} \mathrm{N}$, near $0.55 \mathrm{nbar}(Z p=9)$, with wind speeds approaching $-0.5 \mathrm{kms}^{-1}$ (see Figure 1a). The corresponding meridional wind speeds are likewise equatorward in response to temperature gradients [Bougher et al., 2005; Majeed et al., 2005, 2009], with magnitudes in the range $50 \mathrm{~ms}^{-1}$ to $120 \mathrm{~ms}^{-1}$ for pressures $\leq 0.03 \mu \mathrm{bar}(Z p>5)$ (see Figure $1 \mathrm{~b}$ ). While the magnitudes of vertical wind speeds of $5 \mathrm{~ms}^{-1}$ are upward (see Figure 1c) in the high latitude of both the southern and northern hemispheres, these winds turn downward at low latitudes with magnitudes of $-1 \mathrm{~ms}^{-1}$. Clearly, with the assumed inputs in the JTGCM described above, this simulation of global wind system, which was 
run for 95 Jovian rotations to achieve equilibrium down to $1 \mu$ bar, drives a strong global circulation of neutral flow with strong polar outflows up to $5 \mathrm{~ms}^{-1}$ and convergent flows with vertical wind speeds up to $1 \mathrm{~ms}^{-1}$ near the equator. Such a strong simulated circulation of neutral flow in the polar regions at the $\mu$ bar and nbar levels (see Figure 1) is responsible for the global redistribution of neutral temperatures and composition in the Jovian thermosphere [Bougher et al., 2005].

[27] Similar behavior of neutral flow in the northern hemisphere is simulated by Tao et al. [2009] with westward directed zonal wind speeds up to $0.7 \mathrm{kms}^{-1}$, compared to the JTGCM speeds up to $0.5 \mathrm{kms}^{-1}$, accompanied by equatorward directed meridional wind speeds of $\sim 70 \mathrm{~ms}^{-1}$ near $74^{\circ}$ latitude at the top of the atmosphere. They have also shown the maximum poleward directed meridional wind speeds of approximately a factor of 6 slower in the upper thermosphere than those simulated by this JTGCM simulation near $79^{\circ}$ latitude. This poleward flow combined with polar outflows with vertical wind speeds of $\sim 0.75 \mathrm{~ms}^{-1}$ in the Tao et al. [2009] model effectively cools off the northern highlatitude region up to an altitude of $1800 \mathrm{~km}$. This result is in contradiction with the JTGCM result that shows poleward flow being confined near the ionospheric peak up to an altitude of $600 \mathrm{~km}$ where the Coriolis force is dominant. The calculated low to midlatitude subsidence of neutral flow with vertical wind speeds in the range $\sim 0.2-0.3 \mathrm{~ms}^{-1}$ by Tao et al. is generally in agreement with the JTGCM but with wind speeds larger by a factor of 3 to 5 . 
[28] On the other hand, Smith and Aylward [2009], using baseline model inputs as described above, have shown that diverging neutral winds are generated in the Jovian polar region, resulting from large temperature gradients. These winds are rapidly turned westward by Coriolis forces, causing a strong zonal flow at $\sim 800 \mathrm{~km}$ and an equatorward directed meriodional jet in the region poleward of the main auroral oval connected to different regions in the Jovian magnetodisk. The structure of zonal flow stars to break down in the Jovian oval as the plasma drift abruptly changes direction. In the lower atmosphere near the Jovian ionospheric peak $(\sim 500 \mathrm{~km})$, the westward flow generated by ion drag and Joule heating is deflected by Coriolis force which gives rise to a strong poleward flow in the region close to the main auroral oval similar to that simulated by the JTGCM in the northern oval. However, the high-latitude energy in the baseline model of Smith and Aylward [2009] is not sufficient to be transferred meridionally to the Jovian equatorial regions. This makes the model even more sensitive to extra heat injected either in the polar or equatorial regions. The heat advection from the polar region to the Jovian equator is undermined by the action of Coriolis force that slows down the rotation speed of both the thermosphere and middle magnetosphere, leaving the equatorial regions relatively cooler than the measured temperature. Using the extra heat equatorward of the main oval Smith and Aylward [2009] interpreted the observation of high equatorial temperature. However, at the same time the poleward convection of heat, resulting from induced pressure gradients by equatorial heating raises the polar temperatures close to those inferred from the infrared observations of the Jovian aurora [Lam et al., 1997; 
Stallard et al., 2002; Raynaud et al., 2004]. One of the consequences of this poleward heating is that it tends to rotate the Jovian middle magnetospheric plasma at a much faster rate than observations [McNutt et al., 1979]. The model developed by Smith and Aylward [2009] describes the rotational coupling and angular momentum transfer between the Jovian thermosphere and magnetosphere self-consistently with global neutral dynamics and energy budget. The JTGCM on the other hand is essentially a circulation model describing the global thermal and dynamical structures self-consistently with composition distribution in the Jovian thermosphere by imposing the magnetospheric forcing at the oval locations.

[29] Both Tao et al. [2009] and Smith and Aylward [2009] require an extra source of heating in their models to enhance underlying meridional flow with necessary speeds to reproduce high-latitude temperature observations in addition to Galileo temperature observations near the Jovian equator. However, as shown above, for assumed inputs into the JTGCM a substantial amount of Joule heating is produced in the Jovian ovals. The downward scaling of Joule heating and ion drag is needed in the JTGCM to explain the Jovian thermospheric temperatures observed in both auroral and equatorial regions [see Bougher et al., 2005, Majeed et al., 2005, 2009 for details]. It is also worth noting that in all existing global Jupiter models, an aurorally driven wind system alone cannot generate strong divergent and upwelling flow in the oval regions that must subside and converge at the Jovian equator [e.g., Majeed et al., 2005, 2009; Bougher et al., 2005; Tao et al., 2009;

Smith and Aylward, 2009; Yates et al., 2012, 2014]. 


\subsection{Winds in the Auroral Ovals}

[30] As mentioned above, all existing Jovian models have shown that the highlatitude Joule heating is critical in controlling the neutral temperature in auroral regions. The corresponding process of ion drag and pressure gradients have also been shown to greatly impact the neutral winds in the Jovian thermosphere. These winds are used to

disperse auroral heat across Jupiter and thus controlling the global temperature and associated energy budget. In Figure 2 we show the average vertical profiles of the magnitudes of zonal, meridional, and vertical wind velocities for the northern and southern ovals. These averages have been calculated separately from individual wind profiles corresponding to each grid point in the JTGCM along the oval in each hemisphere. Note that the general behavior of these wind profiles reflects the Jovian wind structure shown in Figure 1. In Figure 2a, it is shown that the zonal winds are blowing predominantly westward in the southern oval peaking at around $0.04 \mu$ bar with a speed of $\sim 1.6 \mathrm{kms}^{-1}$. A rapid increase in the zonal wind speed from $200 \mathrm{~ms}^{-1}$ to $1.5 \mathrm{kms}^{-1}$ is seen between 1 and $0.1 \mu$ bar owing to a combined impact of zonal accelerations resulting from Coriolis forcing, ion drag, a large pressure gradient, and advection processes. In the upper thermosphere $(<0.01 \mu$ bar $)$, the zonal flow is blowing with almost a constant speed of $1.5 \mathrm{kms}^{-1}$.

[31] The vertical distribution of meridional winds for neutral flow in the southern oval has a very different structure. These winds in the lower thermosphere between 1 $\mu$ bar and $0.05 \mu$ bar are blowing poleward of the southern oval with the peak speed of - 
$600 \mathrm{~ms}^{-1}$, consistent with general circulation of neutral flow resulting from Joule heating and the ion-drag process (Figure 1b). The wind speeds for pressures $<0.05 \mu$ bar are reversed and blowing equatorward with speeds up to $300 \mathrm{~ms}^{-1}$. The corresponding vertical winds in the JTGCM are small in magnitude and thus are shown in Figure 2a with a factor of 50 more than their original values. Interestingly, the vertical winds for

pressures $>0.1 \mu$ bar are blowing downward with speeds of nearly $2 \mathrm{~ms}^{-1}$, yielding a neutral motion to compress the lower thermosphere. This compression in the JTGCM yields adiabatic heating of the neutral atmosphere, to be reconciled with hydrodynamic cooling induced by the meridional flow of neutral winds. This has been shown to be an effective method of supplying the net heating rate for the lower thermosphere in the southern oval to obtain neutral temperatures consistent with those derived from multispectral observations. In the upper thermosphere for pressures $<0.1 \mu \mathrm{bar}$, the vertical winds with speeds up to $5 \mathrm{~ms}^{-1}$ are shown to be upward, yielding adiabatic cooling of the neutral atmosphere [Majeed et al., 2009; Bougher et al., 2005].

[32] Figure 2b shows the vertical profiles of the magnitudes of average zonal, meridional, and vertical wind velocities, representing the northern auroral oval in the JTGCM. As expected, the zonal winds are westward throughout the northern oval with a peak speed of $\sim 325 \mathrm{~ms}^{-1}$ near $0.02 \mu$ bar $(\sim 1300 \mathrm{~km})$, consistent with that obtained by Tao et al [2009]. The magnitude of this wind speed is almost a factor of 5 smaller than that for the southern oval owing to underlying hemispheric asymmetries in the ion drag and Joule heating terms, as described by Bougher et al. [2005]. It is important to note that the 
circulation of neutral flow is quite variable between $1 \mu$ bar and 1 nbar. The competing processes in this region give rise to a westward zonal wind speed of about $50 \mathrm{~ms}^{-1}$ at 1 $\mu$ bar while a speed of $\sim 150 \mathrm{~ms}^{-1}$ is obtained at 1 nbar. This speed in the northern oval region at nano-bar levels is about a factor of 5 slower than that obtained by Tao et al. [2009]. The corresponding meridional flow of neutral wind is poleward of the northern " oval with a speed of $\sim 25 \mathrm{~ms}^{-1}$ at $1 \mu$ bar and is found to approach a maximum speed of about $100 \mathrm{~ms}^{-1}$ at $0.5 \mu$ bar. This wind speed steadily decreases to near zero at $\sim 0.04 \mu$ bar and remains at this magnitude until it turns equatorward near $0.01 \mu$ bar. This behavior of meridional wind speeds in the northern oval is quite similar to that simulated by Tao et al. [2009]. Note that the corresponding vertical winds are found to be upward and vary with speeds from $1 \mathrm{~ms}^{-1}$ near $1 \mu$ bar to almost $1.3 \mathrm{~ms}^{-1}$ near $1 \mathrm{nbar}$, with a peak speed of $\sim 4 \mathrm{~ms}^{-1}$ at $0.05 \mu \mathrm{bar}$. For pressures $<1 \mathrm{nbar}$, the simulated wind profiles of neutral flow in

three directions are found to be quite stable with dominant westward (zonal) winds of speeds up to $-225 \mathrm{~ms}^{-1}$ near the top boundary of the JTGCM. The corresponding equatorward meridional wind speeds steadily increase from $-40 \mathrm{~ms}^{-1}$ to around $-75 \mathrm{~ms}^{-1}$ from 1 nbar to the top of the JTGCM boundary, respectively (see Figure 2b), consistent with those simulated by Tao et al. [2009]. However, the vertical wind speeds, which are upward for pressures $<1$ nbar, vary between $1 \mathrm{~ms}^{-1}$ and $2 \mathrm{~ms}^{-1}$ in the northern oval, consistent with the general circulation of the neutral flow described in Figure 1, but somewhat larger than those simulated by Tao et al. [2009]. This upwelling serves to cool off the upper thermosphere of Jupiter, contributing to net heating rate to fit the measured 
exospheric thermal structure [Majeed et al., 2009; Bougher et al., 2005]. Note that the direction of the flow pattern is similar in both ovals, indicating a global meridional circulation cell driven at the ovals which consists of a large pole-to-equator circulation in the upper thermosphere followed by a return flow at the lower thermosphere.

[33] Figure 3 shows the vertical profiles of the magnitudes of neutral horizontal wind (neutral wind hereafter) velocities calculated for the southern and northern ovals from the zonal and meridional components $\left(\sqrt{u^{2}+v^{2}}\right)$ shown in Figure $2 \mathrm{a}$ and $2 \mathrm{~b}$, respectively, to highlight the general characteristics of the Jovian auroral wind system. As described above, the strong westward zonal jet resulting from Joule heating and iondrag processes, combined with the associated meridional flow of neutral winds, predominantly determines neutral winds in the two Jovian oval regions.

[34] The peak in the vertical profile of the neutral wind speed in the northern oval is $\sim 0.3 \mathrm{kms}^{-1}$ near $0.03 \mu \mathrm{bar}$. This value is about a factor of 5.4 smaller than that calculated for the southern oval at about the same pressure. The structure in the neutral wind speeds between $1 \mu \mathrm{bar}$ and $0.01 \mu \mathrm{bar}$ is due to various competing acceleration processes involved in determining the zonal and meridional wind speeds for the two Jovian ovals. These acceleration processes will be discussed in detail in the next section. Note that for pressures $\leq 1 \mu \mathrm{bar}$, the neutral wind speeds simulated in the northern oval regions are almost 4 to 6 times smaller than those simulated in the southern oval regions. This difference in neutral wind speeds reflects hemispheric asymmetry in ion-drag and Joule heating processes resulting from north-south asymmetry in magnetosphere-ionosphere 
mapping using the VIP4 magnetic field model [see Appendix A1]. One of the consequences of this asymmetry is that the process of ion drag becomes significantly more important in the southern hemisphere because of faster ion drifts resulting from magnetosphere-ionosphere coupling [Bougher et al., 2005; see also Millward et al., 2005]. Furthermore, smaller magnitudes of the local magnetic field from VIP4 model in the northern hemisphere compared to those in the southern hemisphere [cf., Connerney et al., 1998] give rise to larger integrated Pedersen conductivities in the southern oval than in the northern oval [see Figure A1 and Bougher et al., 2005]. It is also important to note that geometrical effects that derive larger drifts patterns provide continuous ion drag forcing to the southern oval for a larger portion of the Jovian rotation. In general, it is clear that the underlying thermospheric wind system in the Jovian ovals can be effectively driven by ion drag and Joule heating processes rather than auroral precipitation of energetic charged particle alone [Bougher et al., 2005].

[35] In Figure 4, we compare the JTGCM results of the neutral wind speed with the CFHT wind speed, representing the northern polar region derived from the measured Doppler shifts of infrared $\mathrm{H}_{2}$ lines near $2.1 \mu \mathrm{m}$ [Chaufray et al., 2011]. Only an average $\mathrm{H}_{2}$ wind speed of $0.6 \pm 0.4 \mathrm{kms}^{-1}$ is measured at an estimated height of $\mathrm{H}_{2} \mathrm{~S}_{1}$ line emissions, emanating from a broad range of heights $\sim 700-300 \mathrm{~km}$. This wind speed represents a narrow region of the Jovian aurora at latitudes $\sim 65-75^{\circ}$ and $\lambda_{I I I}$ longitudes $140-180^{\circ}$ mapped by CFHT within the $\mathrm{L}=30 \mathrm{R}_{\mathrm{J}}$ feet of the magnetic tube flux as defined by the VIP4 model. In a recent study of the Jovian infrared aurora of the northern polar 
region, Uno et al. [2014] have accurately estimated the height of the $\mathrm{H}_{2} \mathrm{~S}_{1}$ line emission to be $\sim 640 \pm 90 \mathrm{~km}\left(7 \times 10^{-3}-2.4 \times 10^{-2} \mu \mathrm{bar}\right)$. The average neutral wind speed along with zonal and meridional components is calculated from individual JTGCM profiles, representing an auroral region within the range of latitudes $62-77^{\circ}$ and $\lambda_{\mathrm{III}}$ longitudes 140-180 $0^{\circ}$, covering $\mathrm{H}_{2} \mathrm{~S}_{1}$ emission inside and outside of the main auroral oval. We find that the JTGCM neutral wind speed in the northern auroral region is about $25 \%$ slower than the absolute value of the CFHT wind speed as shown in Figure 4. However, the lower limit of the CFHT wind speed is within the JTGCM speed at a pressure level where the meridional flow is poleward and decreasing as strong Coriolis force tends to deviate it to flow westward with a maximum zonal wind speed of $-450 \mathrm{~m} / \mathrm{s}$ (see Figure 4).

\subsection{Auroral Accelerations}

[36] Jupiter's thermosphere has been proven to be a great heat engine driven by charged particle precipitation and magnetospheric forcing in many studies as mentioned above. The heat inputs from these sources in the JTGCM produce large localized temperature (pressure) gradients and drive considerable neutral wind. The vertical velocity accompanied by this wind is critically important for the Jovian energetics, causing cooling in auroral ovals via upwelling and divergence while heating in the nonauroral latitudes via subsidence and convergence [cf., Bougher et al., 2005; Majeed et al., 2005, 2009]. This pole-to-equator flow of heat is a direct consequence of Joule heating and enhanced ion neutral momentum coupling in the polar region. The neutral wind velocity resulting from temperature gradients depends on the Coriolis force due to 
Jupiter's rotation and ion drag due to ion neutral momentum coupling in the presence of strong magnetic field. According to Rishbeth [1998], the ratio of Coriolis force to ion drag determines the wind direction for the case of steady-state neutral wind. If ion drag is the dominant force as in the case of the Jovian oval near the ionospheric peak then the wind will be blowing parallel to temperature gradients - that is the heat flow could be meridional. However, the larger Coriolis force than ion drag will blow the wind perpendicular to temperature gradients - that is the heat flow will be directed zonally. When both ion drag and Coriolis force are significant and in competition, the wind will be inclined to temperature gradients at an angle and the heat flow would have zonal and meridional components in two-dimensional plane. The thermal balances in the southern and northern oval regions have been shown to be dominated by strong ion drag and Joule heating processes which greatly strengthen the global Jovian wind system to maintain and derive enormous variations in the observed thermospheric temperatures in the polar region [Smith and Aylward 2009; Tao et al., 2009; Bougher et al., 2005; Majeed et al., 2005; 2009]. The corresponding dynamical balance that derives the zonal and meridional components of the neutral wind in the Jovian ovals is described by competing acceleration terms defined in the zonal and meridional momentum equations.

[37] We have calculated the vertical profiles of competing acceleration terms, originating from various physical processes that take place in the Jovian thermosphere. These terms are used to explain the height distribution of zonal and meridional winds responsible for driving the neutral flow in the southern and northern ovals. The 
individual acceleration terms averaged over the Jovian northern and southern ovals are shown in Figures 5 and 6, respectively. Note that we refer to positive zonal terms as eastward forcing and negative zonal terms as westward forcing. The positive and negative meridional terms in the northern oval are referred to as poleward forcing and equatorward forcing, respectively. However, in the southern oval the positive meridional $=$

terms are referred to as equatorward forcing and negative meridional terms are referred to as poleward forcing.

[38] Figure 5a shows the vertical profiles of zonal acceleration of neutral flow generated by various competing processes in the southern Jovian oval. It can be seen that, for pressures $<1$ nbar, the competition between eastward forcing resulting from Coriolis plus advection processes and a combined westward forcing due to large pressure gradients (geopotential gradient forcing) and curvature processes predominantly determines the distribution of zonal winds in the southern oval (see Figure 2a). The eastward forcing due to Coriolis force (see Figure 5a) becomes the dominant process, compared to all other processes in the region between $1 \mathrm{nbar}$ and $0.1 \mu \mathrm{bar}$, to explain advancing zonal flow that peaks at around $0.03 \mu \mathrm{bar}$, with the peak wind speed of $\sim 1.6$ $\mathrm{kms}^{-1}$ (see Figure 2a). However, for pressures $>0.1 \mu$ bar, eastward Coriolis forcing alone competes with westward forcing generated by the ion-drag and other minor (curvature, advection, and geopotential gradient) processes. The maximum in the main eastward and westward forcing terms occurs at about $0.45 \mu \mathrm{bar}$, with magnitudes of $0.25 \mathrm{~ms}^{-2}$ and -0.14 $\mathrm{ms}^{-2}$, respectively. The balance between these forcing terms (with minor components 
from other terms) gives rise to a net acceleration of the neutral flow with a peak value of $\sim 0.055 \mathrm{~ms}^{-2}$ near $0.45 \mu$ bar (see Figure 5a). This net acceleration serves to increase zonal wind speed from the lower boundary of the JTGCM to $0.1 \mu$ bar (see Figure 2a).

[39] Figure 5b shows the calculated vertical profiles of competing meridional forcing terms from various processes for the southern oval. Clearly, as can be seen in Figure 5b,

the cause of meridional flow of neutral winds in the thermosphere of the southern oval is primarily determined by the competition between strong poleward forcing due to Coriolis force plus a small contribution of poleward forcing from the ion drag process, and corresponding equatorward forcing, resulting from large pressure gradients in addition to small contributions from curvature and advection processes. The magnitude of the maximum poleward forcing due to Coriolis force is $\sim 0.37 \mathrm{~ms}^{-2}$ at $0.02 \mu \mathrm{bar}$, while the magnitude of corresponding equatorward forcing is varied from $\sim 0.2 \mathrm{~ms}^{-2}$ to $\sim 0.3 \mathrm{~ms}^{-2}$ for

pressures $<0.02 \mu$ bar. These large magnitudes of meridional accelerations indicate that meridional winds in the southern oval must be very large (see Figure 2a) to play an active role in circulating neutral flow in the southern oval regions. Figure $5 \mathrm{~b}$ also shows the vertical profile of the net magnitude of meridional acceleration determined from various processes with a peak of about $0.17 \mathrm{~ms}^{-2}$ near $0.3 \mu \mathrm{bar}$. The variation in the magnitude of the net meridional acceleration can be used to interpret changes in meridional wind speeds. As can be seen in Figure 5b, an increasing magnitude of net acceleration from the lower boundary of the JTGCM to $\sim 0.5 \mu$ bar is responsible for strengthening the poleward flow of neutral winds to provide dynamical heating, while the weakening of 
this poleward flow for pressures $<0.5 \mu$ bar (see Figure 2a) can be explained by decreasing magnitudes of net acceleration.

[40] As discussed above, the underlying hemispheric asymmetries in ion drag and Joule heating processes in the Jovian system yield smaller magnitudes up to a factor of 5 of momentum forcing terms in order to explain the zonal and meridional winds in the $=$ northern oval compared to those in the southern oval. The vertical profiles of these forcing terms from various processes are shown in Figures $6 \mathrm{a}$ and $6 \mathrm{~b}$. The competition between eastward forcing due to large pressure gradients and a combined westward forcing due to Coriolis force and hydrodynamic process is primarily responsible for driving the zonal flow of neutral winds in the northern oval for pressures $<0.1 \mu$ bar. However, in the lower thermosphere $(>0.1 \mu \mathrm{bar})$, eastward forcing of neutral flow due to Coriolis force alone competes with westward forcing due to ion-drag process and becomes an important source in the JTGCM to explain the simulated zonal winds in the northern oval. Figure 6a shows the magnitudes of the maximum eastward and westward forcing of approximately $0.03 \mathrm{~ms}^{-2}$ and $0.015 \mathrm{~ms}^{-2}$ at $\sim 0.4 \mu \mathrm{bar}$, due to Coriolis force and ion-drag forcing, respectively. The magnitudes of these forcing terms in the upper thermosphere for pressures $<0.1$ nbar vary by about $50-60 \%$ of their values near 0.4 $\mu \mathrm{bar}$, in order to maintain zonal flow with wind speeds in the northern oval shown in Figure 2b. Clearly, the net zonal flow in the lower thermosphere of the northern oval simulated by the JTGCM is dominated by Coriolis forcing, while forcing caused by pressure gradients (zonal gradients) largely contributes to the net zonal flow in the upper 
thermosphere (see Figure 6a). The general trend of variation in net acceleration reflects the general behavior of the zonal flow of neutral winds in the thermosphere of the northern oval (see Figure 2b).

[41] Note that the JTGCM magnitudes of the westward momentum forcing terms in the northern Jovian oval are larger than those calculated by Tao et al. [2009] for latitudes $=$ averaged over $65^{\circ}-80^{\circ} \mathrm{N}$. The reason for this difference in zonal magnitudes is recently explained by Tao et al. [2014], who claimed that both the energy and momentum transport equations did not include the effect of zonal gradient terms in their earlier model [see Tao et al., 2009]. By ignoring the effects of these terms in transport equations, the zonal distribution of high-latitude heat at Jupiter's thermosphere increases the local temperatures continuously until large-scale convection cells are generated. These cells are used to transport excess heat globally through meridional processes and winds. Tao et al. [2014] have compared the Jovian thermospheric simulations both with and without the effects of zonal gradient terms in transport equations. They found that the effect of zonal gradient terms can reduce the neutral wind speeds as much as a factor of $\sim 6$ compared to those simulated by ignoring the effect of zonal gradient terms. This is the reason the magnitudes of the westward directed zonal wind speeds at auroral altitudes simulated by the JTGCM are $\sim 30 \%$ slower than those simulated by the Tao et al.'s model.

[42] In Figure $6 \mathrm{~b}$ we show the vertical profiles of meridional accelerations of neutral flow averaged over the northern oval from different competing processes along with the 
net acceleration to understand the general behavior of meridional winds in the northern thermosphere. Clearly, poleward Coriolis torque acting on anticorotational flow in the oval region plays a significant role compared to other competing processes in generating poleward meridional flow that peaks at $\sim 0.03 \mu \mathrm{bar}$, with a magnitude of $\sim 0.05 \mathrm{~ms}^{-2}$. A fractionally small poleward forcing $\left(<-0.01 \mathrm{~ms}^{-2}\right)$ due to ion drag process is calculated near $1 \mu \mathrm{bar}$. However, small equatorward forcing of neutral flow is also calculated for pressure $>0.01 \mu$ bar by other processes, such as advection, curvature, and pressure gradients. Thus, among all the possible processes in the JTGCM, poleward forcing of neutral flow by the Coriolis force for pressure $\geq 0.01 \mu$ bar is the dominant process in maintaining the neutral winds in the northern oval thermosphere (see Figure $2 b$ ) and transporting heat in that region [Bougher et al., 2005; Smith and Aylward, 2009]. However, an increase in equatorward forcing of neutral flow is calculated primarily from pressure gradients in the pressure range from $0.01 \mu$ bar to the upper boundary of the JTGCM. This equatorward forcing is in competition with poleward Coriolis forcing for pressure $<0.01 \mu$ bar. Similar behavior of meridional forcing terms was simulated by Tao et al. [2009], but with larger magnitudes than the JTGCM because of the reason mentioned above. The competition between equatorward and poleward forcing profiles from various source terms in the JTGCM gives rise to a net forcing profile with a poleward directed peak value of about $0.02 \mathrm{~ms}^{-2}$ near $0.5 \mu \mathrm{bar}$. The strength of this net forcing gradually becomes weaker and weaker until it turns equatorward for pressures less than a few nbar, reflecting meridional flow in the JTGCM with wind speeds 
described by Figure 2b. This equatorward flow in the JTGCM contributes to the net cooling of the exospheric region of the northern oval [Bougher et al., 2005; Majeed et al., 2009].

[43] While we are currently in the process of developing a self-consistent coupled atmosphere-thermosphere-magnetosphere model called the Jupiter - Global Ionosphere " Thermosphere Model (J-GITM) to study the Jovian system, improved wind measurements are also needed to reconcile instrumental errors and to constrain the altitude and temporal variations observed in and around the Jovian oval.

\section{Summary}

[44] We show that the JTGCM, using auroral heating produced by processes of particle precipitation and substantial amount of Joule heating, produced by frictional motions of ions and neutrals in the Jovian ovals can generate sufficient magnitudes of zonal and meridional winds, in addition to vertical winds at Jupiter needed to describe the Jovian dynamics. We also show that redistribution of these winds in the Jovian thermosphere is driven by various acceleration processes that take place in the Jovian ovals. The net acceleration, dominated by Coriolis forcing in the lower thermosphere $(>0.1 \mu$ bar), drives the average zonal flow of neutral winds in both the southern and northern ovals, with the peak magnitudes of $\sim 1.6 \mathrm{kms}^{-1}$ and $\sim 0.65 \mathrm{kms}^{-1}$, respectively. The average zonal flow for pressures $<0.01 \mu$ bar in the two ovals is predominantly determined by net acceleration produced by geopotential gradient forcing compared to Coriolis forcing. The minor accelerations generated from hydrodynamic advection, 
curvature, and viscosity processes are also shown to contribute to the net acceleration for the upper Jovian thermosphere. We find that the average meridional winds of neutral flow in the lower thermosphere $(>0.01 \mu$ bar $)$ in both ovals is poleward and is mainly controlled by net acceleration caused by Coriolis forcing. We also find that the magnitude of this net acceleration in the southern oval is about a factor of 3 larger than in the $=$ northern oval. This difference in net acceleration appears to be due to a hemispheric asymmetry in magnetic fields. The poleward flow of meridional winds, combined with subsiding flow resulting from vertical winds, provides significant heat to the oval regions. We find that the strength of the poleward flow of neutral winds in both ovals starts to weaken at around $0.5 \mu$ bar and eventually turns equatorward near $0.01 \mu$ bar. This behavior is consistent with decreasing net acceleration for pressures $<0.5 \mu$ bar. We also find that the average neutral wind speeds in the southern oval regions are more than a factor of 4 - 7 greater than these in the northern oval regions. Clearly, this imbalance in wind speeds reflects a north-to-south asymmetry in the JTGCM circulation caused by Joule heating and ion-drag processes related to hemispheric differences in high-latitude ion drifts, VIP4 magnetic field topologies, and Pedersen conductivities.

[45] A comparison of the vertical profiles of average neutral wind speed is also made with $\mathrm{H}_{2}$ wind speed recently derived from CFHT observations of the Jovian northern aurora confined to a narrow region of $65-75^{\circ}$ latitude and $140-180^{\circ}$ longitude. We find that the modeled wind speed in the northern oval is approximately $25 \%$ smaller than that observed with CHFT. This difference between the JTGCM and observed winds appears 
to be due to both instrumental uncertainties in measuring the $\mathrm{H}_{2}$ winds and the approximate nature of the JTGCM input parameterizations. Clearly, new observations of neutral winds both in the northern and southern ovals of Jupiter are warranted. JUNO observations of the Jovian system will provide evidence of corotation speeds of neutrals and ions in the magnetosphere-ionosphere coupling to validate wind speeds simulated by current models.

Appendix A: Parameterizations of Convection Electric Field, Ion Drag and Joule heating

\section{A1. Convection electric field model}

[46] A simplified magnetosphere-ionosphere coupling model based on Voyager measurements of ion convection perpendicular to the magnetic field in the outer magnetosphere is developed to calculate magnetospheric potentials that are mapped to

the ionosphere and are used to derive convection electric fields and corresponding ion drifts. The VIP4 magnetic field model [Connerney et al., 1998] is employed to map this Jovian magnetospheric potential pattern to ionospheric heights. The basic equation to be solved to determine the convection velocity of the Jovian magnetospheric plasma at the auroral latitudes is cf., Cravens and Gombosi, 2004]:

$$
\mathbf{V}=\frac{\mathbf{E X B}}{\left|B^{2}\right|}
$$

[47] In the magnetosphere, the bulk velocity of plasma flow is related to electric field via the generalized form of Ohm's law [Cravens and Gombosi, 2004]:

$$
\mathbf{E}=-\mathbf{V}_{\Phi} \mathbf{x} \mathbf{B}
$$


where $\mathbf{V}_{\boldsymbol{\Phi}}$ is the bulk plasma velocity vector, while $\mathbf{B}$ is the VIP4 magnetic field vector in the Jovian magnetosphere. The Voyager observations of the Jovian inner magnetosphere have shown that the plasma is in fairly rigid corotation with the neutral upper atmosphere out to a radial distance of $16 \mathrm{R}_{\mathrm{J}}$ with a speed of $200 \mathrm{kms}^{-1}$ [McNutt et al., 1981]. A gradual increase in the magnitude of $\mathbf{V}_{\boldsymbol{\Phi}}$ is observed in the middle magnetosphere until an equilibrium speed of $\sim 500 \mathrm{kms}^{-1}$ is reached near $50 \mathrm{R}_{\mathrm{J}}$ [Carbary et al., 1981]. Eviatar and Barbosa [1984] assumed this constant flow of the Jovian magnetospheric plasma in the outer magnetosphere up to $70 \mathrm{R}_{\mathrm{J}}$ in their model to describe the importance of heavy ions of Iogenic origin in an attempt to explain the observed auroral radiation power on Jupiter. The measured speed of plasma flow by Voyager in the three regions of the Jovian magnetosphere as a function of $\mathrm{R}_{\mathrm{J}}$ is shown in Figure A1. This speed is used in equation 11 to estimate the convection electric field in the Jovian magnetosphere. The electric field is integrated in the radial direction to derive an axisymmetric magnetospheric potential. This potential is mapped to the Jovian high-latitude ionosphere using the VIP4 magnetic field model, and the derivative is taken to provide the electric field that points towards the equator as viewed in a corotating frame of reference. Using this electric field coupled with the VIP4 magnetic field lines in the polar ionosphere we estimate plasma drift vectors in the Jovian high-latitude region, which are counter to the corotation in both the northern and southern hemispheres. Figure 8 of Bougher et al. [2005] illustrated this anticorotational ion flow and relative ion wind vectors which are pronounced and well organized in both hemispheres. The magnitudes of zonal ion winds 
in both ovals approach $3 \mathrm{kms}^{-1}$, while meridional ion winds are almost half of these values. It is found that the plasma drifts in the southern polar region are about $20 \%$ stronger than those in the northern polar region owing to the mapping of VIP4 magnetic fields. Clearly, this results in a larger departure of the southern polar drifts from corotation than the polar drifts in the north. The estimates of anticorotational ion drift

vectors are assumed to vary in space, but are constant in time with speed up to $3 \mathrm{kms}^{-1}$ in the zonal direction and up to $1.5 \mathrm{kms}^{-1}$ in the meridional directions [Bougher et al., 2005]. These drift vectors are parameterized in the JTGCM on a two dimensional (zonal and meridional) grid to investigate the global dynamics of the neutral flow in the Jovian upper thermosphere. They are also used to calculate ion drag parameters and Joule heating self-consistently in the JTGCM by using the above formulations.

\section{A2. Ion Drag and Joule Heating}

[48] The ionospheric ions within the auroral regions are constrained by their motion along the magnetic field lines while electric fields are used to accelerate these ions having collisions with slowly moving neutrals. In this process ions lose their momentum and dragging the neutrals in their same direction. Thus the strength of ion drag strongly depends on the magnitude of the localized electric and magnetic fields and is derived by the relative drift caused by the number of ion-neutral collisions which are expected to be large near the ionospheric peak [Roble et al., 1981]. The relative drift between ions and neutrals in this process is the primary cause of Joule heating within the Jovian auroral ovals. Ion drag is an important process in the JTGCM that drives the global dynamics of 
the neutral flow. We parameterized ion-drag and Joule heating in the JTGCM code according to the formulation described by Roble and Ridley [1987] with the following ion-drag parameters called tensors for the $10^{\circ}$ offset Jovigraphic and Jovimagnetic poles.

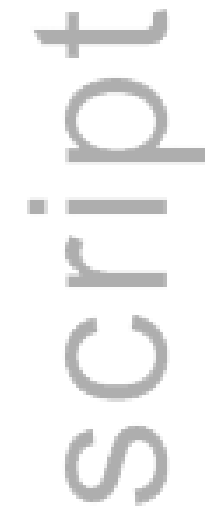

$$
\begin{gathered}
\alpha_{x x}=\alpha_{1}\left(1-\sin ^{2} \delta \cos ^{2} I\right) \\
\alpha_{y y}=\alpha_{1}\left(1-\cos ^{2} \delta \cos ^{2} I\right) \\
\alpha_{x y}=\alpha_{1} \sin \delta \cos \delta \cos ^{2} I+\alpha_{2} \sin I \\
\alpha_{y x}=\alpha_{1} \sin \delta \cos \delta \cos ^{2} I+\alpha_{2} \sin I
\end{gathered}
$$

where $\delta$ and I are the magnetic declination and magnetic dip angles, respectively, and

$$
\begin{aligned}
& \alpha_{1}=\frac{\sigma_{P} B^{2}}{\rho} \\
& \alpha_{2}=\frac{\sigma_{H} B^{2}}{\rho}
\end{aligned}
$$

where $\sigma_{\mathrm{p}}$ represents the Pedersen conductivity while Hall conductivity is represented by $\sigma_{\mathrm{H}} \cdot \mathrm{B}$ is the strength of the magnetic field from the VIP4 model [Connerney et al., 1998], and $\rho$ is the JTGCM neutral density. Figure 1A shows the average vertical profile of the Pedersen and Hall conductivities in the northern and southern oval regions. The maximum values of these conductivities near the Jovian ionospheric peak are one and two orders of magnitudes larger than those obtained by Smith and Aylward [2009] and Tao et al. [2009], respectively, by using larger values of auroral inputs, VIP4 magnetic 
field model, and faster plasma drifts velocities (see Table 1). The Joule heating in the JTGCM is derived from the ion-drag parameters and frictional motion between ions and neutrals as [cf., Roble and Ridley, 1981]

$$
Q_{J}=\alpha_{x x}\left(u_{i}-u_{n}\right)^{2}+\alpha_{y y}\left(v_{i}-v_{n}\right)^{2}+\left(\alpha_{x y}-\alpha_{y x}\right)\left(u_{i}-u_{n}\right)\left(v_{i}-v_{n}\right)
$$

where $\mathrm{u}_{\mathrm{i}}$ and $\mathrm{v}_{\mathrm{i}}$ are the jovigraphic zonal and meridional components of ion drift $=$

velocities inputted from a simple magnetospheric convection model described above in section A1. $\mathrm{u}_{\mathrm{n}}$ and $\mathrm{v}_{\mathrm{n}}$ are the JTGCM zonal and meridional neutral wind components determined at a given time step.

[49] Acknowledgements. We are grateful to the National Center for Atmospheric Research (NCAR) for the use of the IBM/SP and SGI supercomputer resources necessary to develop and exercise the JTGCM thermospheric model and its postprocessor. In addition, special thanks go to Ben Foster of NCAR for his help preparing the JTGCM code to run on the IBM/SP computers. This work is supported by NASA/STScI grant HST-AR-09941.01-A, NASA/Planetary Atmosphere grant NNG0SGB40G, and NASA grant NNX13AC77G through subcontract X13-7142-UM to the University of Michigan. TM also acknowledges Faculty Research Grant from the research office at AUS. The models used in this study will be made available upon request by TM. We also thank the two anonymous reviewers for their critical reviewing of this paper thoroughly and making useful comments and suggestions to improve the quality of the original submission. 


\section{References}

Achilleos, N., S. Miller, J. Tennyson, A. D. Aylward, I. Mueller-Wodarg, and D. Rees (1998), JIM: A time-dependent, three-dimensional model of Jupiter's thermosphere $=$ and ionosphere, J. Geophys. Res., 103, 20,089-20,112.

Bougher, S. W., J. H. Waite, T. Majeed and G. R. Gladstone (2005), Jupiter Thermosphere General Circulation Model (JTGCM): Global studies and dynamics driven by auroral and Joule heating, J. Geophys. Res.,110, E04008, doi:10.1029/2003JE002230.

Broadfoot, A. L., et al. (1979), Extreme ultraviolet observations from Voyager 1 encounter with Jupiter, Science, 204, 979.

Carbary, J. F., et al. (1981), Ion anisotropies in the outer Jovian magnetosphere, J. Geo. Res., 86, 8285-8300.

Chaufray, J.-Y., G. R. Gladstone, J. H. Waite, and J. T. Clake, (2010), Asymmetry in the Jovian auroral Lyman- $\alpha$ line profil due to thermospheric high-speed flow, J. Geophys. Res., 115, E05002, doi: 10.1029/2009JE003439.

Chaufray, J.-Y., et al. (2011), Spectro-imaging observations of Jupiter's $2 \mu \mathrm{m}$ auroral emission. II Thermospheric winds, Icarus, 211, 1233-1241. 
Connerney, J. E. P., M. H. Acuna, N. F. Ness, and T. Satoh (1998), New models of Jupiter's magnetic fields constrained by the Io flux tube footprint, J. Geophys. Res., 103, 11,929-11,940.

Cowley, S. W. H., and E. J. Bunce (2001), Origin of the main auroral oval in Jupiter's coupled magnetosphere-ionosphere system, Planet. Space Sci., 49, 1067-1088.

Cowley, S. W. H., E. J. Bunce, and J. D. Nichols (2003a), Origins of Jupiter's main auroral oval emissions, J. Geophys. Res., 108, doi:10.1029/2002JA009329.

Cowley, S. W. H., E. J. Bunce, T. S. Stallard, and S. Miller (2003b), Jupiter's polar ionospheric flows: Theoretical interpretation, Geophys. Res. Lett., 30, 1220, doi:10.1029/2002GL016030.

Cowley, S. W. H., et al. (2005), A simple axisymmetric model of magnetosphereionosphere coupling currents in Jupiter's polar ionosphere, J. Geophys. Res., 110, A11209, doi: 10.1029/2005JA011237.

Cowley, S. W. H., A. J. Deason, E. J. Bunce (2008), Axi-symmetric models of auroral current systems in Jupiter's magnetosphere with predictions for the Juno mission, Ann. Geophys.,26, 4051-4074.

Cravens, T. E. (1987), Vibrationally excited molecular hydrogen in the upper atmosphere of Jupiter, J. Geophys. Res. 92, 11,083-11,100, doi:10.1029/JA092iA10p11083.

Cravens, T. E., J. H. Waite, T. I. Gombosi, N. Lugaz, G. R. Gladstone, B. H. Mauk, and R. J. MacDowall (2003), Implications of Jovian X-ray emission for ionospheremagnetosphere coupling, J. Geophys. Res., SMP 25, doi:10.1029/2003JA010050. 
Cravens, T. E., and T. I. Gombosi (2004), Cometary magnetospheres: a tutorial, $A d v$.

Space Res., 33, 1968-1976

Dickinson, R. E., E. C. Ridley, and R. G. Roble (1981), A three-dimensional general circulation model of the thermosphere, J. Geophys. Res., 86, 1499.

Drossart, P., et al. (1989), Detection of $\mathrm{H}_{3}{ }^{+}$on Jupiter, Nature, 340, 539-541.

Drossart, P., B. Bezard, S. K. Atreya, J. Bishop, J. H. Waite, Jr., and D. Boice (1993), Thermal profiles in the auroral regions of Jupiter, J. Geophys. Res., 98, 18,80318,811 .

Elsner, R. F., et al. (2005), Simultaneous Chandra X ray, Hubble Space Telescope ultraviolet, and Ulysses radio observations of Jupiter's aurora, J. Geophy. Res., 110, A01207, doi:1029/2004JA010717.

Eviatar, A., and A. D. Barbosa (1984), Jovian magnetospheric neutral wind and auroral precipitation flux, J. Geophys. Res., 89, 7393.

Festou, M. C., et al. (1981), Composition and thermal profiles of the Jovian upper atmosphere determined by the Voyager steller occultation experiment, $J$. Geophys.Res., 86, 5715-5735.

Frank, L. A., and W. R. Paterson (2001), Survey of thermal ions in the Io plasma torus with the Galileo spacecraft, J. Geophys. Res., 106, 6131-6150.

Gérard, J.-C., D. Grodent, A. Rodioti, B. Bonford, and J. T. Clark (2013), Hubble observations of Jupiter's north- south conjugate ultraviolet aurora, Icarus, 226, 15591567. 
Gérard, J.-C., J. Gustin, D. Grodent, J. T. Clark, and A. Grard (2003), Spectral observations of transient features in the FUV Jovian polar aurora, J. Geophys. Res., 108, doi: 10.1029/2003JA009901.

Gladstone, G. R., J. H. Waite Jr., and J.-C. Gérard (1998), Jovian auroral Lyman-a self-

reversals: A window on Jupiter's auroral electrojet?, Bull. Am. Astron. Soc., 30, 1078.

= Gladstone, G. R., et al. (2002), A pulsating auroral X-ray hot spot on Jupiter, Nature, $415,1000-1003$.

Grodent, D., J. H. Waite, Jr., and J-C. Gerard (2001), A self-consistent model of the Jovian auroral thermal structure, J. Geophys. Res., 106, doi:10.1029/200JA900129.

Grodent, D., J. T. Clarke, J. Kim, J. H. Waite, and S. W. H. Cowley (2003), Jupiter's main auroral oval observed with HST-STIS, J. Geophys. Res., 108, 1389, doi:10.1029/2003JA009921.

Grodent, D., J.-C, Gérard, A. Radioti, B. Bonfond, and A. Saglam (2008), Jupiter's changing auroral location, J. Geophys. Res., 113, A01206, doi:10.1029/2007JA012601.

Gustin, J., et al. (2004a), Jovian auroral spectroscopy with FUSE: Analysis of selfabsorption and implications for electron precipitation, Icarus, 171, 336-355.

Gustin, J., J.-C. Gérard, D. Grodent, S. W. H. Cowley, J. T. Clarke, and A. Gérard (2004b), Energy-flux relationship in the FUV Jovian aurora deduced from HST-STIS spectral observations, J. Geophy. Res., 109, A10205, doi: 10.1029/2003JA010365.

This article is protected by copyright. All rights reserved. 
Gustin, J., S. W. H. Cowley, J.-C. Gérard, G. R. Glandstone, D. Grodent, and J. T. Clarke, (2006), Characteristics of Jovian morning bright FUV aurora from Hubble Space Telescope/Space Telescope Imaging Spectrograph imaging and spectral observations, J. Geophy. Res., 111, A09220, doi: 10.1029/2006JA011730.

Hill, T. W. (2001), The Jovian auroral oval, J. Geophys. Res., 106, 8101-8108.

Hill, T. W., and V. M. Vasyliunas (2002), Jovian auroral signatutr of Io's corotational wake, J. Geophys. Res., 107, 1464, doi: 10.1029/2002JA009514.

Kane, M., B. H. Mauk, E. P. Keath, and S. M. Krimigis (1995), Hot ions in Jupiter's magnetodisc: A model for Voyager-2 low-energy charged particle measurements, $J$. Geophys. Res., 100, 19,473-19,486.

Kharchenko, V., A. Dalgarno, D. R. Schultz, and P. C. Stancil (2006), Ion emission spectra in the Jovian X-ray aurora, Geophys. Res. Lett., 33, L11105, doi: 10.1029/2006GL026039.

Khurana, K. K., (2001), Influence of solar wind on Jupiter's magnetosphere deduced from currents in the equatorial plane, J. Geophys. Res., 106, 25,999-26,016.

Kim, S. J. (1988), Infrared processes in the Jovian auroral zone, Icarus, 75, 399-408.

Krupp, N., A. Lagg, S. Livi, B. Wilken, J. Woch, E. C. Roelof, and D. J. Williams (2001), Global flows of energetic ions in Jupiter's equatorial plane: First-order approximation, J. Geophys. Res., 106, 26,017-26,032.

Lam, H. A., et al. (1997), A baseline spectroscopic study of the infrared aurora of Jupiter, Icarus, 127, 379-393. 
Majeed, T., J. H. Waite, S. W. Bougher, and G. R. Gladstone (2005), Processes of equatorial thermal structure at Jupiter: An analysis of the Galileo temperature profile with a three-dimensional model, J. Geophys. Res., 110, E12007, doi:10.1029/2004JE002351.

Majeed, T., J. H. Waite, S. W. Bougher, and G. R. Gladstone (2009), Processes of auroral $=$ thermal structure at Jupiter: Analysis of multispectral temperature observations with the JTGCM, J. Geophys. Res., 114, E07005, doi:10.1029/2008JE003194.

McNutt, R. L., J. W. Belcher, J. D. Sullivan, F. Bagenal, and H. S. Bridge (1979), Departure from corotation of plasma in Jupiter's dayside magnetosphere, Nature, $280,803$.

McNutt, R. L., J. W. Belcher, and H. S. Bridge (1981), Positive ion observations in the middle magnetosphere of Jupiter, J. Geo. Res., 86, 8319-8342.

Miller, S., N. Achilleos, G. E. Ballester, H. A. Lam, J. Tennyson, T. R. Geballe, and L. M. Trafton (1997), Mid-to-low latitude $\mathrm{H}_{3}{ }^{+}$emission from Jupiter, Icarus, 130, 57-67. Millward, G. H., S. Miller, T. Stallard, N. Achilleos, and A. D. Aylward (2005), On the dynamics of the Jovian ionosphere and thermosphere. IV. Ion-neutral coupling, Icarus, 173, 200-211.

Nichols, J. D., and S. W. H. Cowley (2004), Magnetosphere-ionosphere coupling currents in Jupiter's middle magnetosphere: Effect of precipitation-induced enhancement of the ionospheric Pedersen conductivity, Ann. Geophys. 22, 17991827. 
Nichols, J. D., J. T. Clarke, J. C. Gérard, D. Grodent, K. C. Hansen (2009), Variation of different components of Jupiter's auroral emission, J. Geophys. Res., 114, A06210, doi:10.1029/2009JA014051.

Prangé, R., D. Rego, L. Pallier,L. Ben Jaffel, C. Emerich, J. T. Clarke, G. E. Ballester, and J. M. Ajello (1997), Detection of self-reversed Lyman $\alpha$ lines from the Jovian aurorae with the Hubble Space Telescope, Astrophys.J., 484, L169.

Pryor, W., et al. (2005), Cassini UVIS observations of Jupiter's auroral variability, Icarus, $178,312-326$.

Radioti, A., J.-C. Gérard, D. Grodent, B. Bonfond, N. Krupp, and J. Woch (2008), Discontinuity in Jupiter's main auroral oval, J. Geophys. Res., 113, A01215, doi: 10.1029/2007JA012610.

Radioti, A., et. al., (2009), Equatorward diffuse auroral emissions at Jupiter: Simultaneous HST and Galileo observations, Geophys. Res. Lett, L07101, doi:10.1029/2009GL037857.

Ray, L. C., N. A. Achilleos, M. F. Vogt, and J. N. Yates (2014), Local time variations in Jupiter's magnetosphere-ionosphere coupling system, J. GeoPhys. Res., 119, 47404751, doi:10.1002/2014JA019941.

Raynaud, E., et al. (2003), The 10 October 1999 HIP 9369 occultation by the northern polar region of Jupiter: ingress and egress lightcurves analysis, Icarus, 162, 344-361.

Raynaud, E., et al. (2004), Spectro-imaging observations of Jupiter's 2- $\mu \mathrm{m}$ auroral emission, Part I. H3+ distribution and temperature, Icarus, 171, 133-152. 
Rego, D., N. Achilleos, T. Stallard, S. Miller, R. Prangé, M. Dougherty, and R.D. Joseph (1999), Supersonic winds in Jupiter's aurorae, Nature 399, 121-124.

Rishbeth, H (1998), How the thermospheric circulation affects the ionospheric F2-layer, J. Amos. Sol-terr. Phys., 60, 1385-1402.

Roble, R. G., and E. C. Ridley (1987), An auroral model for the NCAR thermospheric $=$ general circulation model (TGCM), Ann. Geophysicae, 5A, 369-382.

Roble, R. G., E. C. Ridley, A. D. Richmond, and R. E. Dickinson (1988), A coupled thermospheric-ionospheric general circulation model, Geophys. Res. Lett., 15, 13251328.

Satoh, T., and J. E. P. Connerney (1999), Jupiter's H3+ emission viewed in corrected Jovimagnetic coordinates, Icarus, 141, 236-252.

Seiff, A., et al. (1998), Thermal structure of Jupiter's atmosphere near the edge of a 5- $\mu \mathrm{m}$ hot spot in the north equatorial belt, J. Geophys. Res., 103, 22,857-22,890.

Smith, C. G. A., and A. D. Ayward (2008), Coupled rotational dynamics of Saturn's thermosphere and magnetosphere: A thermospheric modeling study, Ann. Geophys. $26,1007-1027$.

Smith, C. G. A., A. D. Aylward, G. H. Millward, S. Smith, and L. E. Moore (2007), An unexpected cooling effect in Saturn's upper atmosphere, Nature, 445, 399-401.

Smith, C. G. A., and A. D. Ayward (2009), Coupled rotational dynamics of Jupiter's thermosphere and magnetosphere, Ann. Geophys. 27, 199-230. 
Sommeria, J., L. Ben-Jaffel, and R. Prange (1995), On the existence of supersonic jets in the upper atmosphere of Jupiter, Icarus, 119, 2-24.

Stallard, T., S. Miller, G. Millward, and R. D. Joseph (2001), On the dynamics of the Jovian ionosphere and thermosphere, Icarus, 154, 475-491.

Stallard, T., S. Miller, G. Millward, and R. D. Joseph (2002), On the dynamics of the $=$ Jovian ionosphere. II. The measurements of $\mathrm{H}_{3}{ }^{+}$vibrational temperature, column density and total emission, Icarus, 156, 498-514.

Stallard, T., S. Miller, S. W. H. Cowley, and E. J. Bunce (2003), Jupiter's polar ionospheric flow: Measured intensity and velocity variations poleward of the main auroral oval, Geophys. Res. Lett., 30, 25-1, 25-4.

Tao, C., Y. Miyoshi, N. Achilleos, and H. kita (2014), Response of the Jovian thermosphere to variations in solar EUV flux, J. Geophys. Res., 119, 3664-3682, doi:10.1002/2013JA019411.

Tao, C., H. Fujiwara, and Y. Kasaba (2009), Neutral wind control of the Jovian magnetosphere-ionosphere current system, J. Geophys. Res., 114, A08307, doi:10.1029/2008JA013966.

Uno, T., Y. Kasaba, C. Tao, T. Sakanoi, M. Kagitani, S. Fujisawa, H. Kita, and S. V. Badman (2014), Vertical emissivity profiles of Jupiter's northern H3+ and H2 infrared auroras observed by Subaru/IRCS, J. Geophys. Res., 119, 10,219-10,241, doi:10.1002/2004JA020454. 
Vogt., M. F., et. al. (2011), Improved mapping of Jupiter's auroral features to magnetospheric sources, J. Geophys. Res., 116, A03220, doi:10.1029/2010JA016148.

Waite, J. H., Jr., and D. Lummerzheim (2002), Comparison of auroral processes: Earth and Jupiter, in Atmospheres in the Solar System: Comparative Aeronomy, Geophys. Monogr. Ser., vol. 130, edited by M. Mendillo, A. F. Nagy, and J. H. Waite, pp. 115$=$ 139, AGU, Washington, D. C.

Waite, J. H., Jr., T. E. Cravens, J. U. Kozyra, A. F. Nagy, S. K. Atreya, and R. H. Chen (1983), Electron precipitation and related aeronomy of the Jovian thermosphere and ionosphere, J. Geophys. Res., 88, 6143-6163.

Waite, J. H., Jr., et al. (2000), Multispectral observations of Jupiter's aurora, Adv. Space Res., 26, 1453-1475.

Waite, J. H., Jr., et al. (2001), An auroral flare at Jupiter, Nature, 410, 787-789.

Yates, J. N., N. Achilleos, and P. Guio (2014), Response of the Jovian thermosphere to a transient pulse in solar wind pressure, Planet. Space Sci., 91, 27-44.

Yates, J. N., N. Achilleos, and P. Guio (2012), Influence of upstream solar wind on thermospheric flows at Jupiter, Planet. Space Sci., 61, 15-31.

\section{Captions:}

Figure 1: JTGCM simulation with charged particle heating and the total Joule heating produced in the Jovian ovals. Contours of longitudinal average over the entire JTGCM vertical domain (in log pressure coordinates) are shown for (a) neutral zonal winds (u) in 
$100 \mathrm{~m} \mathrm{~s}^{-1}$ intervals, (b) neutral meridional winds (v) in $50 \mathrm{~m} \mathrm{~s}^{-1}$ intervals, and (c) neutral vertical winds $(\mathrm{w})$ in $1.0 \mathrm{~m} \mathrm{~s}^{-1}$ intervals.

Figure 2: JTGCM simulated profiles of zonal (u), meridional (v), and vertical (w) wind speeds from $20 \mu$ bar to $10^{-4}$ nbar for (a) southern auroral oval and (b) northern auroral oval. Note that original values of the vertical wind speeds are multiplied by a factor of 50 to fit in the figure.

Figure 3: JTGCM simulated profiles of the neutral wind speeds from $20 \mu$ bar to $10^{-4}$ nbar for the northern oval (solid curve) and southern oval (dotted curve).

Figure 4: A comparison of $\mathrm{CFHT}_{2}$ wind speed is shown with the JTGCM simulation of neutral horizontal wind speed averaged over a latitude range $62^{\circ}-77^{\circ} \mathrm{N}$ and system $I I I$ longitude range $140^{\circ}-180^{\circ}$. The corresponding zonal and meridional components of the horizontal wind velocity are also shown.

Figure 5: JTGCM simulated profiles of (a) zonal and (b) meridional acceleration terms from different processes for the southern oval. The corresponding net zonal and meridional acceleration profiles are also shown.

Figure 6: JTGCM simulated profiles of (a) zonal and (b) meridional acceleration terms from different processes for the northern oval. The corresponding net zonal and meridional acceleration profiles are also shown.

Figure 1A: (a) Radial profile of corotation plasma flow speed in three regions of Jupiter's magnetosphere is shown: inner magnetosphere $\left(0-16 \mathrm{R}_{\mathrm{J}}\right)$ with a constant speed 
of $200 \mathrm{~km} \mathrm{~s}^{-1}$, middle magnetosphere $\left(16-50 \mathrm{R}_{\mathrm{J}}\right)$ with speeds between 200 and $500 \mathrm{~km}$ $\mathrm{s}^{-1}$, and outer magnetosphere $\left(50-70 \mathrm{R}_{\mathrm{J}}\right)$ with constant speed of $500 \mathrm{~km} \mathrm{~s}^{-1}$. (b) The vertical profiles of the JTGCM Pedersen and Hall conductivities averaged over the northern and southern ovals.

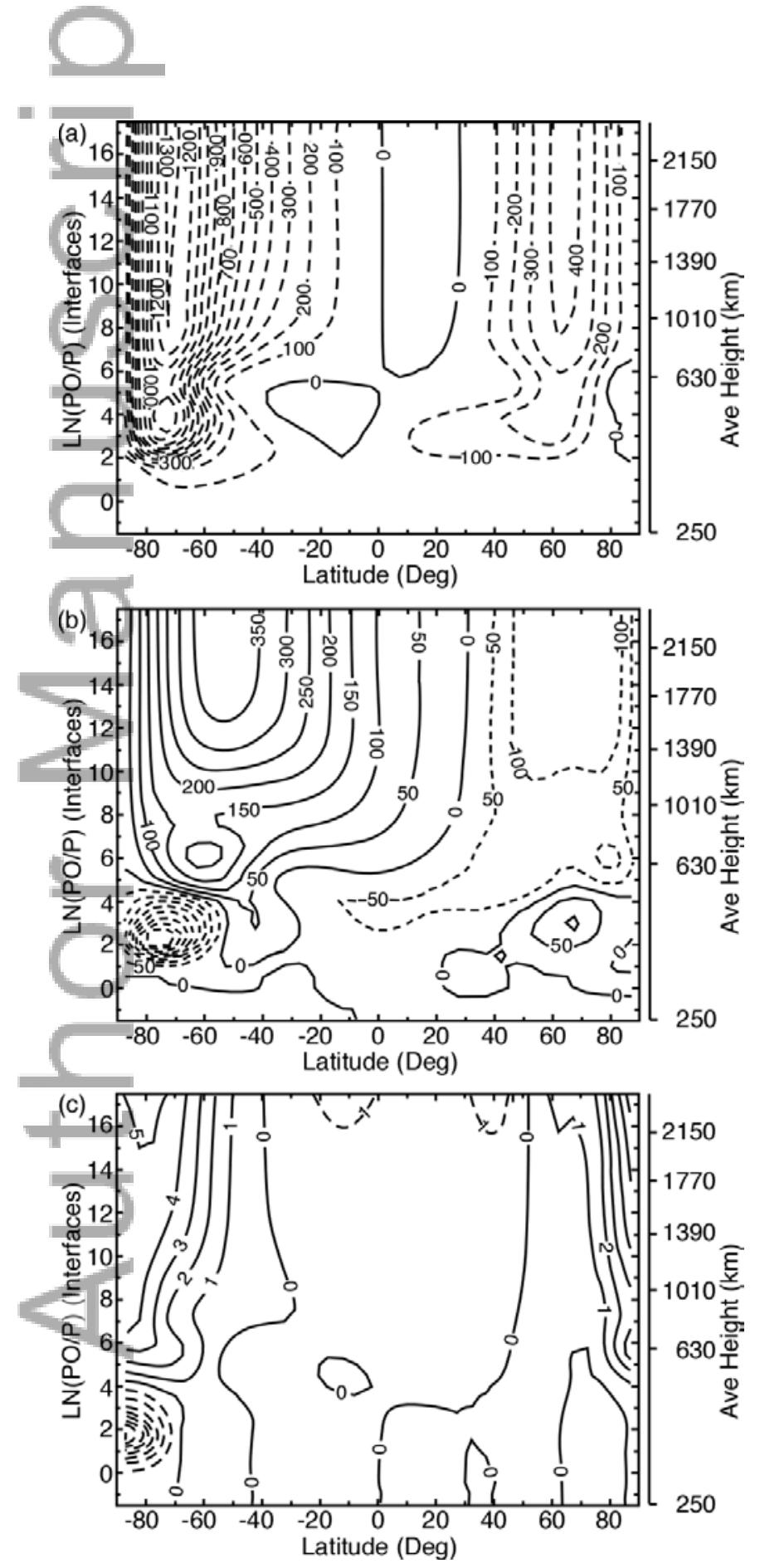

This article is protected by copyright. All rights reserved. 
Figure 1: JTGCM simulation with charged particle heating and the total Joule heating produced in the Jovian ovals. Contours of longitudinal average over the entire JTGCM vertical domain (in - $\log$ pressure coordinates) are shown for (a) neutral zonal winds (u) in $100 \mathrm{~m} \mathrm{~s}^{-1}$ intervals, (b) neutral meridional winds (v) in $50 \mathrm{~m} \mathrm{~s}^{-1}$ intervals, and (c) neutral vertical winds (w) in $1.0 \mathrm{~m} \mathrm{~s}^{-1}$

= intervals.

(

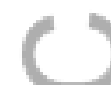

( )
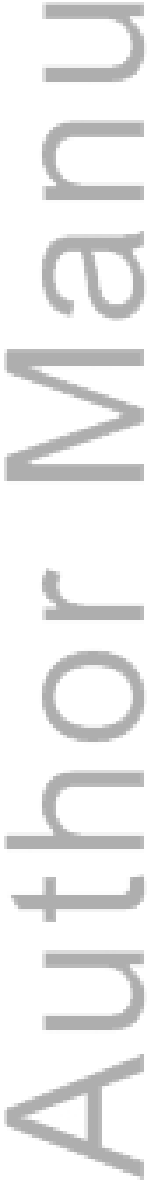

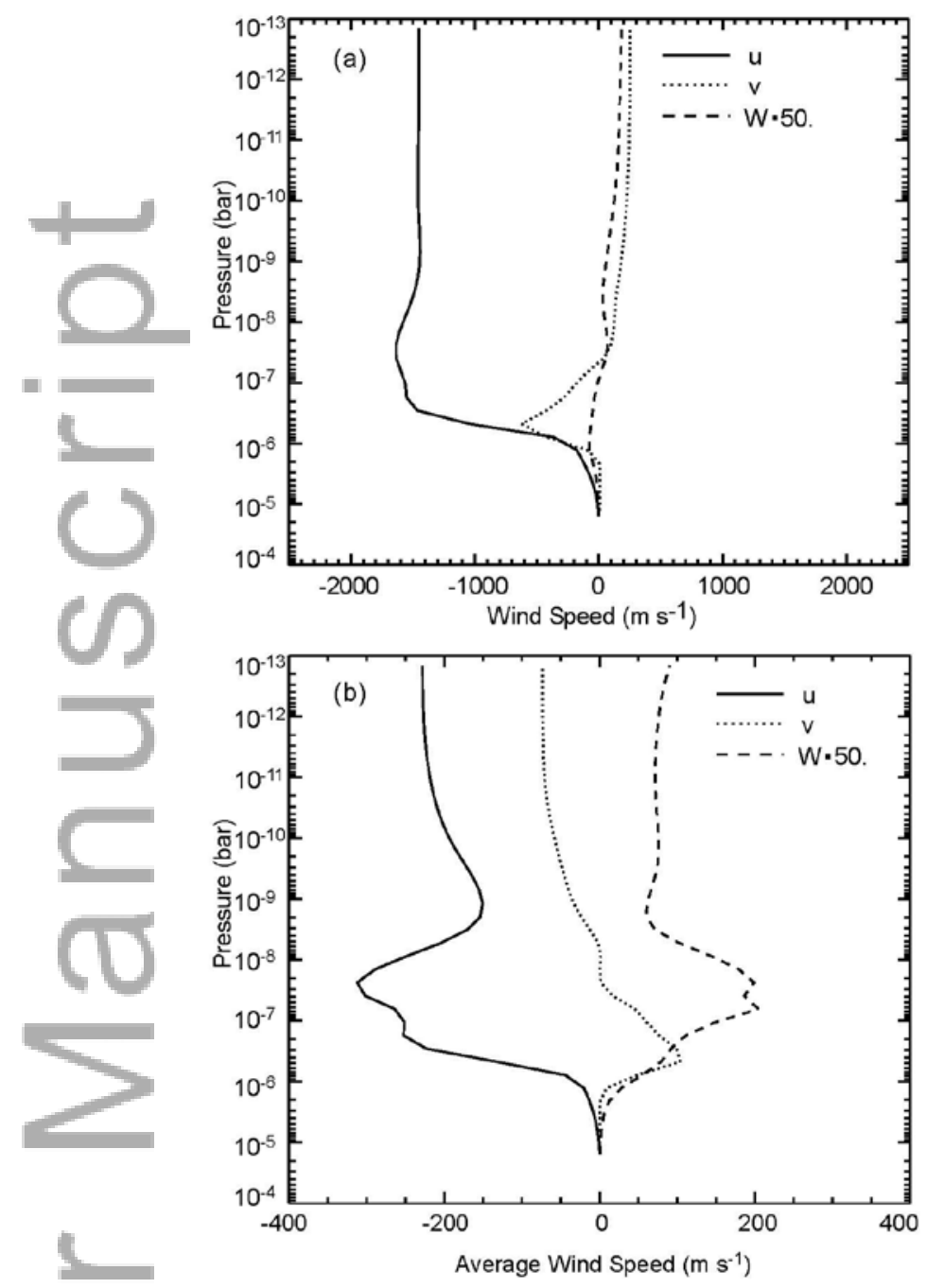

Figure 2: JTGCM simulated profiles of zonal (u), meridional (v), and vertical (w) wind speeds from $20 \mu$ bar to $10^{-4}$ nbar for (a) southern auroral oval and (b) northern auroral oval. Note that original values of the vertical wind speeds are multiplied by a factor of 50 to fit in the figure. 

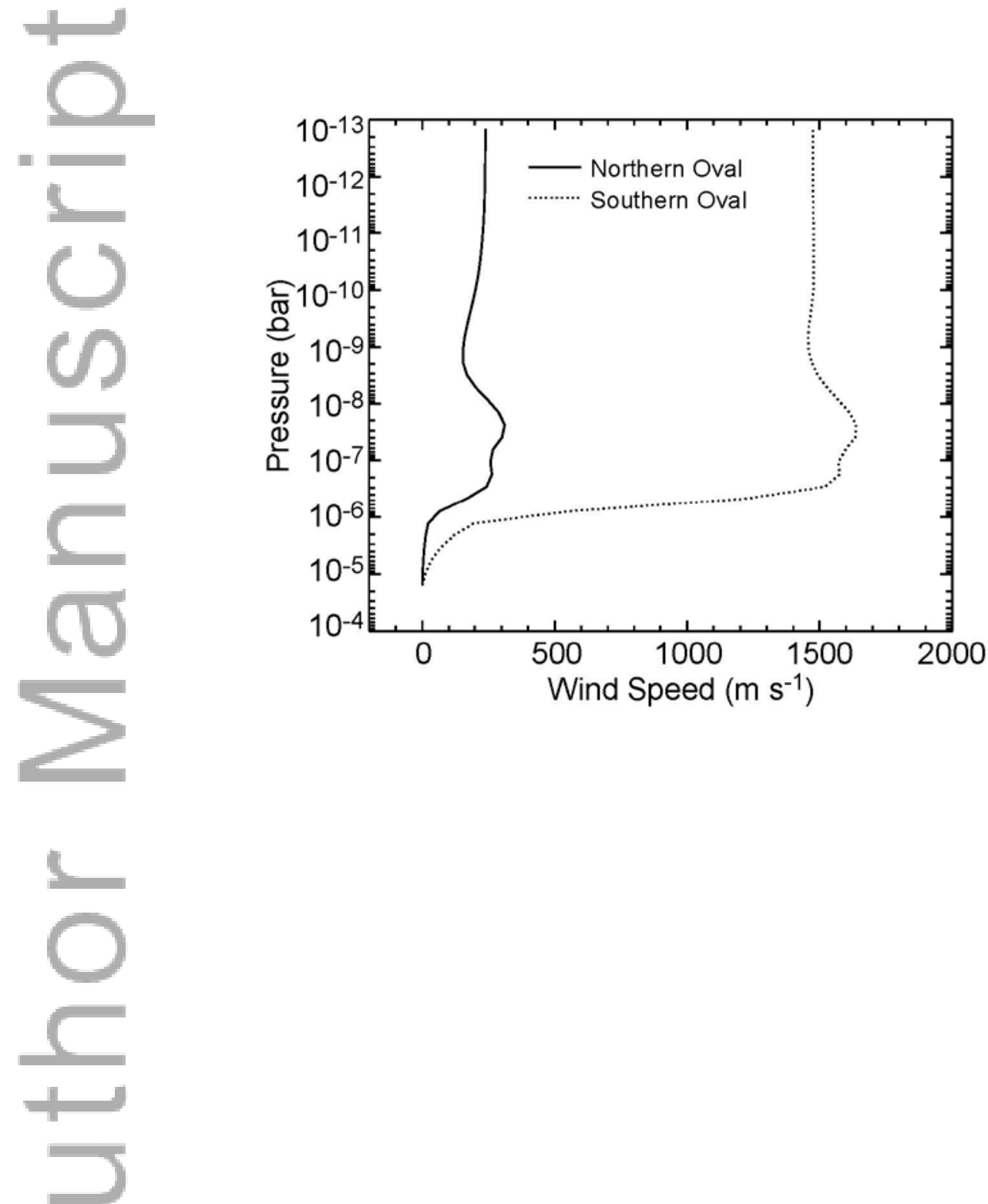

Figure 3: JTGCM simulated profiles of the neutral wind speeds from $20 \mu$ bar to $10^{-4}$ nbar for the northern oval (solid curve) and southern oval (dotted curve). 


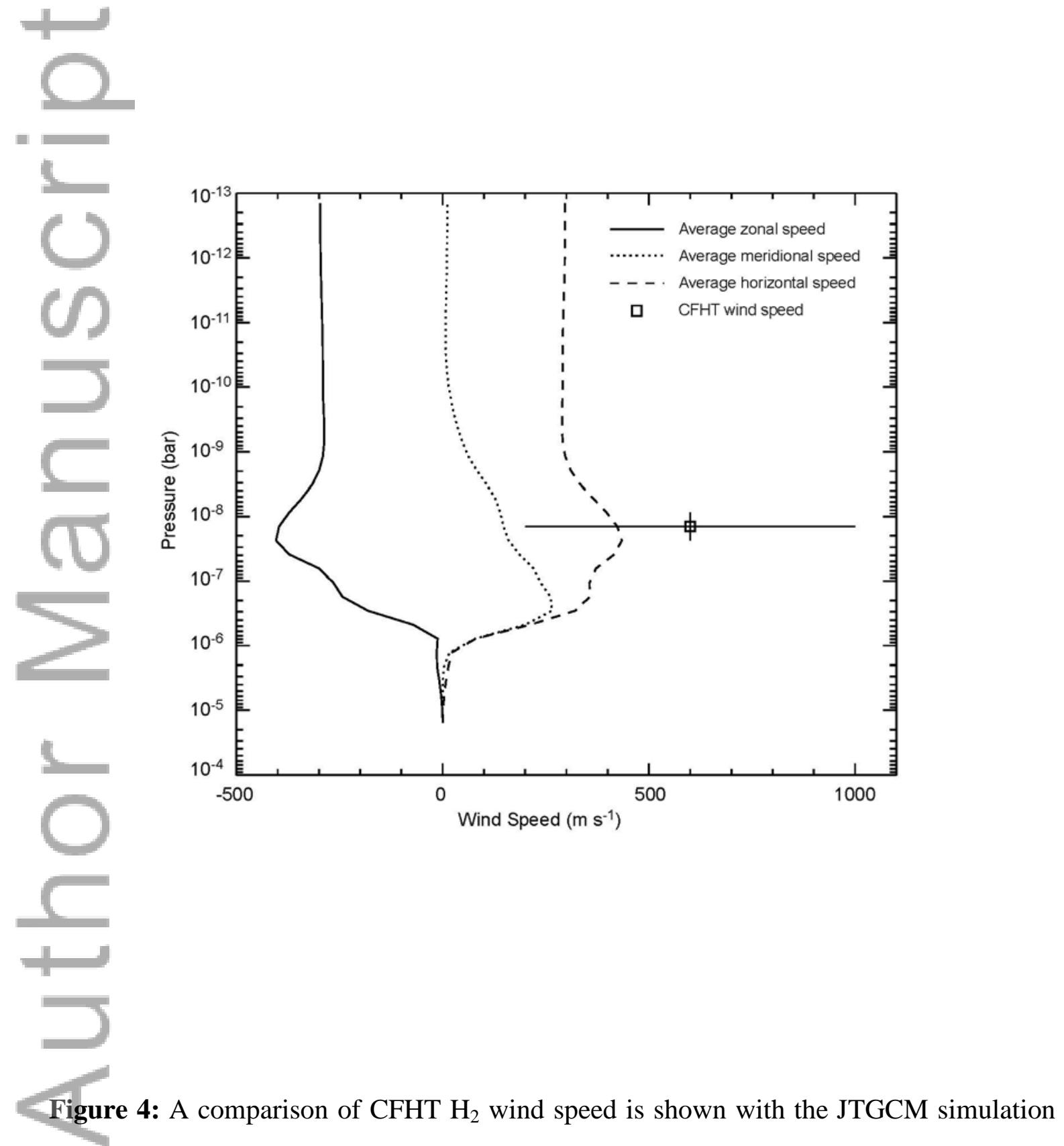

This article is protected by copyright. All rights reserved. 
of neutral horizontal wind speed averaged over a latitude range $62^{\circ}-77^{\circ} \mathrm{N}$ and system III longitude range $140^{\circ}-180^{\circ}$. The corresponding zonal and meridional components of the horizontal wind velocity are also shown.

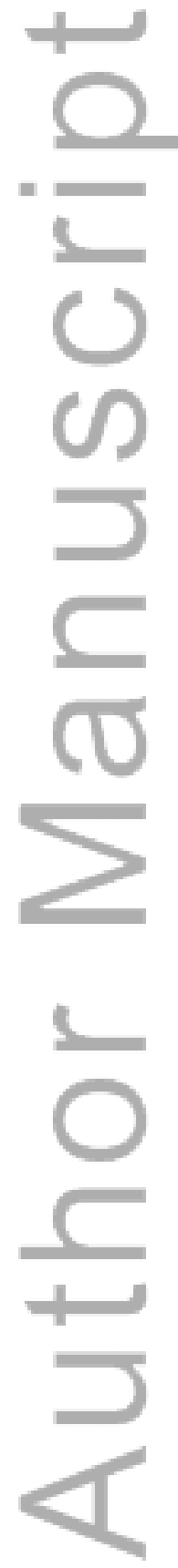



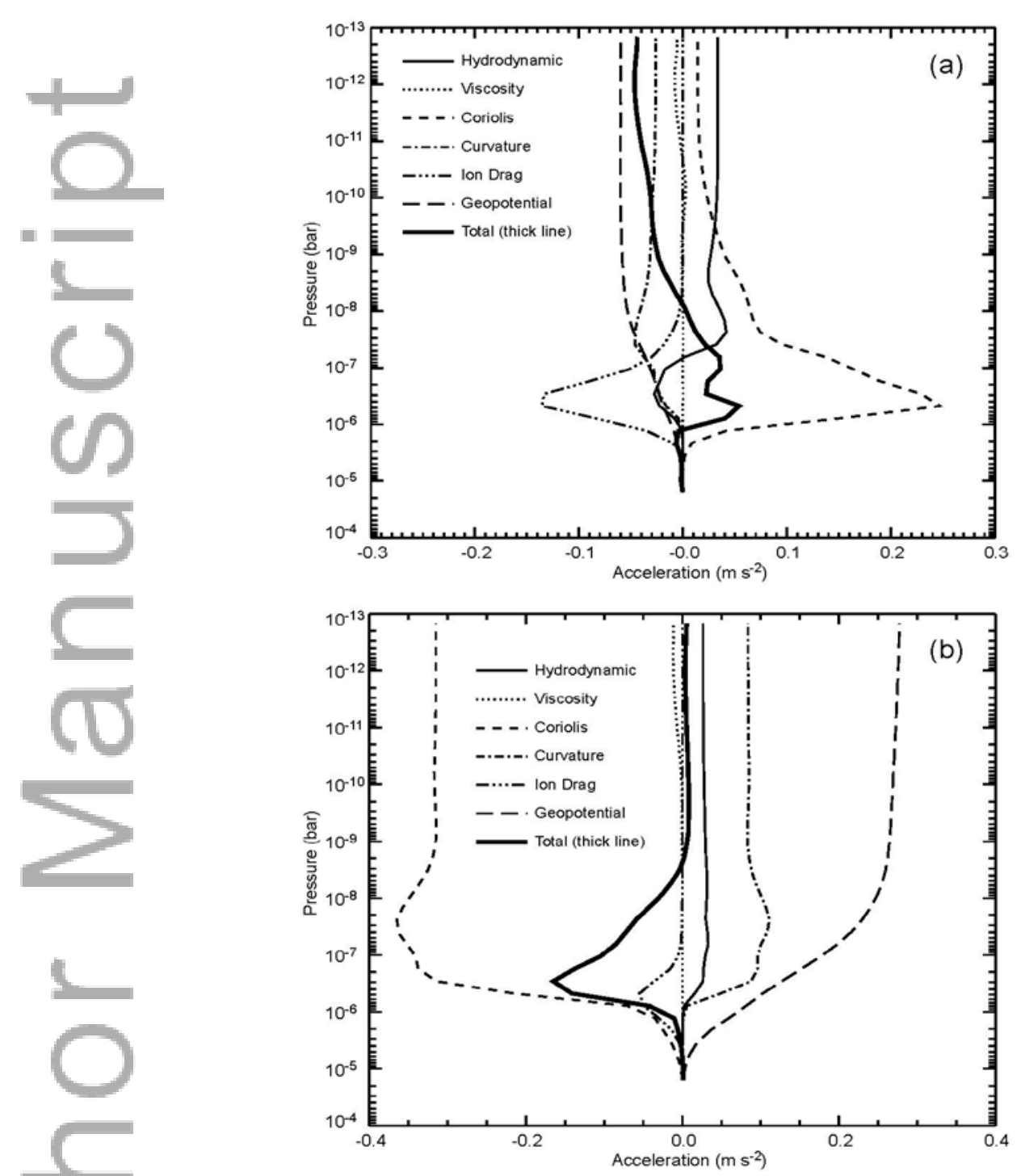

Figure 5: JTGCM simulated profiles of (a) zonal and (b) meridional acceleration terms 
from different processes for the southern oval. The corresponding net zonal and meridional acceleration profiles are also shown.

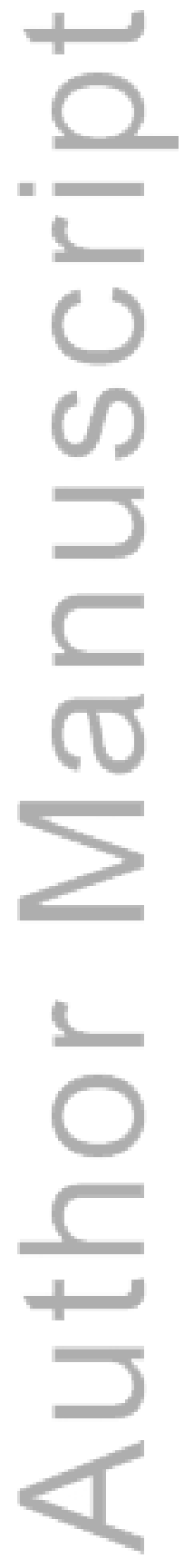



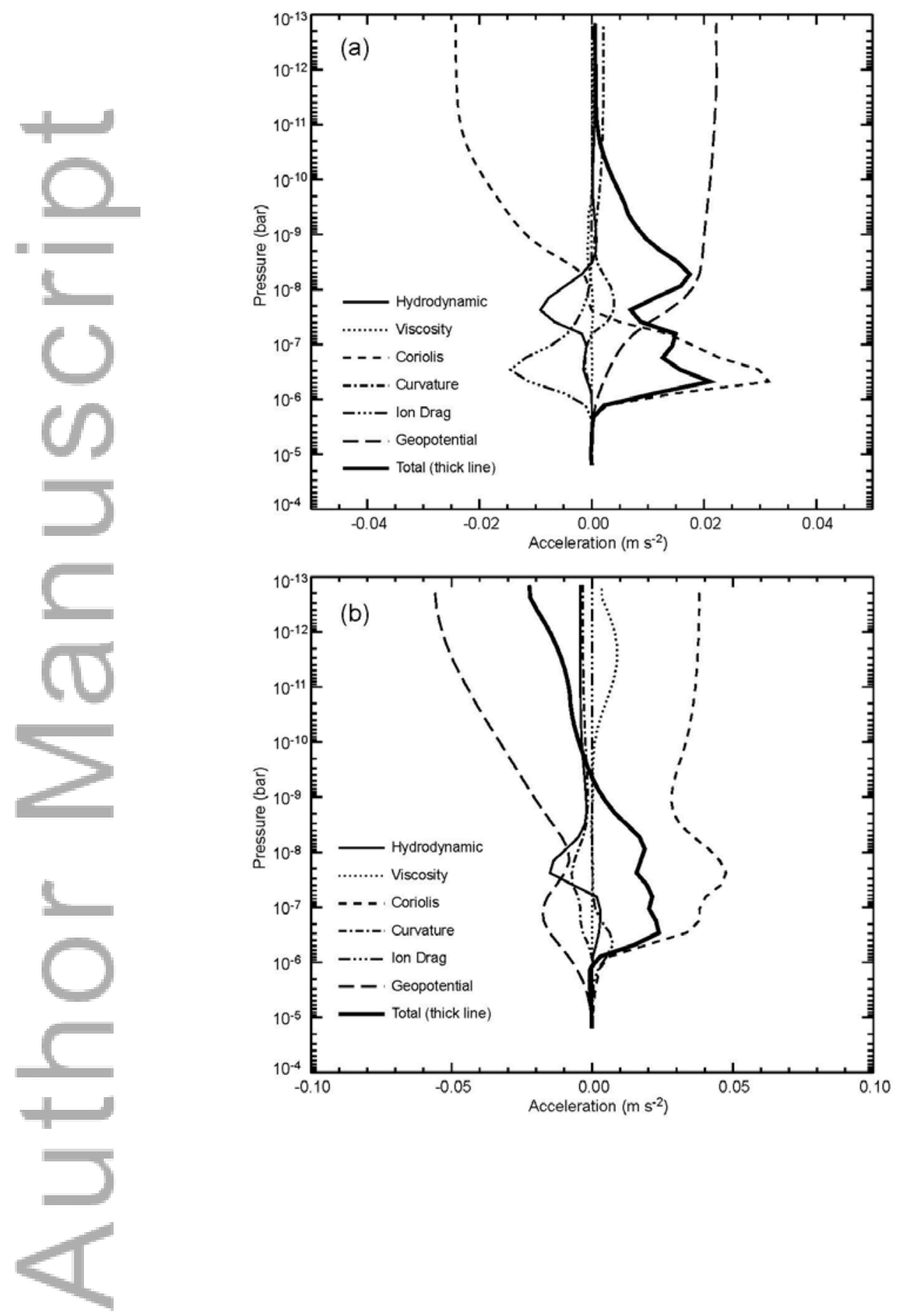
Figure 6: JTGCM simulated profiles of (a) zonal and (b) meridional acceleration terms from different processes for the northern oval. The corresponding net zonal and meridional acceleration profiles are also shown.

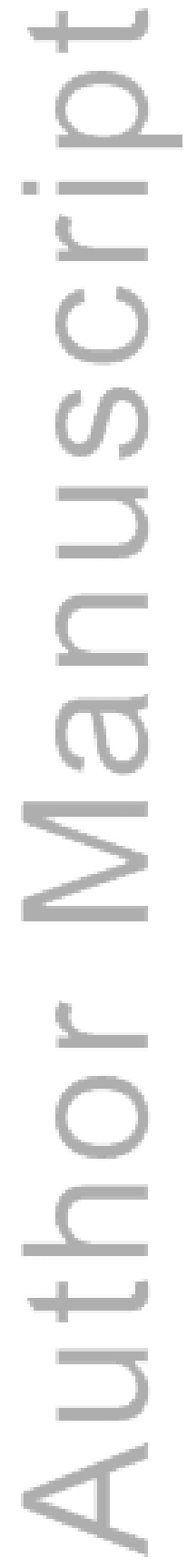



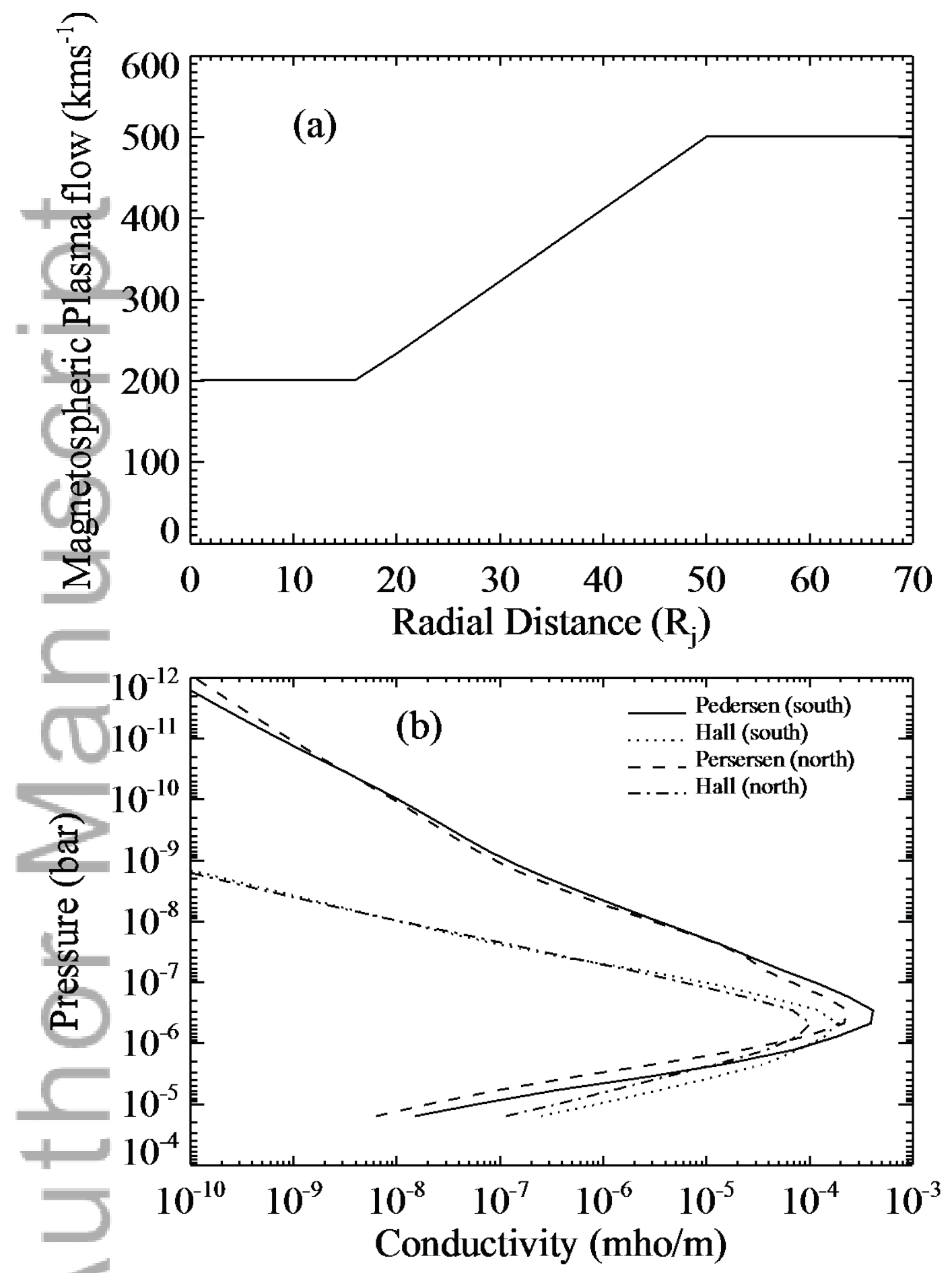

This article is protected by copyright. All rights reserved. 
Figure 1A: (a) Radial profile of corotation plasma flow speed in three regions of Jupiter's magnetosphere is shown: inner magnetosphere $\left(0-16 \mathrm{R}_{\mathrm{J}}\right)$ with a constant speed of $200 \mathrm{~km} \mathrm{~s}^{-1}$, middle magnetosphere $\left(16-50 \mathrm{R}_{\mathrm{J}}\right)$ with speeds between 200 and $500 \mathrm{~km}$ $\mathrm{s}^{-1}$, and outer magnetosphere $\left(50-70 \mathrm{R}_{\mathrm{J}}\right)$ with constant speed of $500 \mathrm{~km} \mathrm{~s}^{-1}$. (b) The vertical profiles of the JTGCM Pedersen and Hall conductivities averaged over the northern and southern ovals.

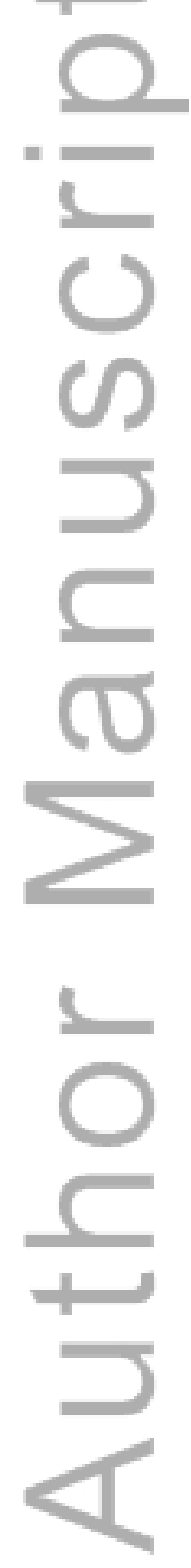



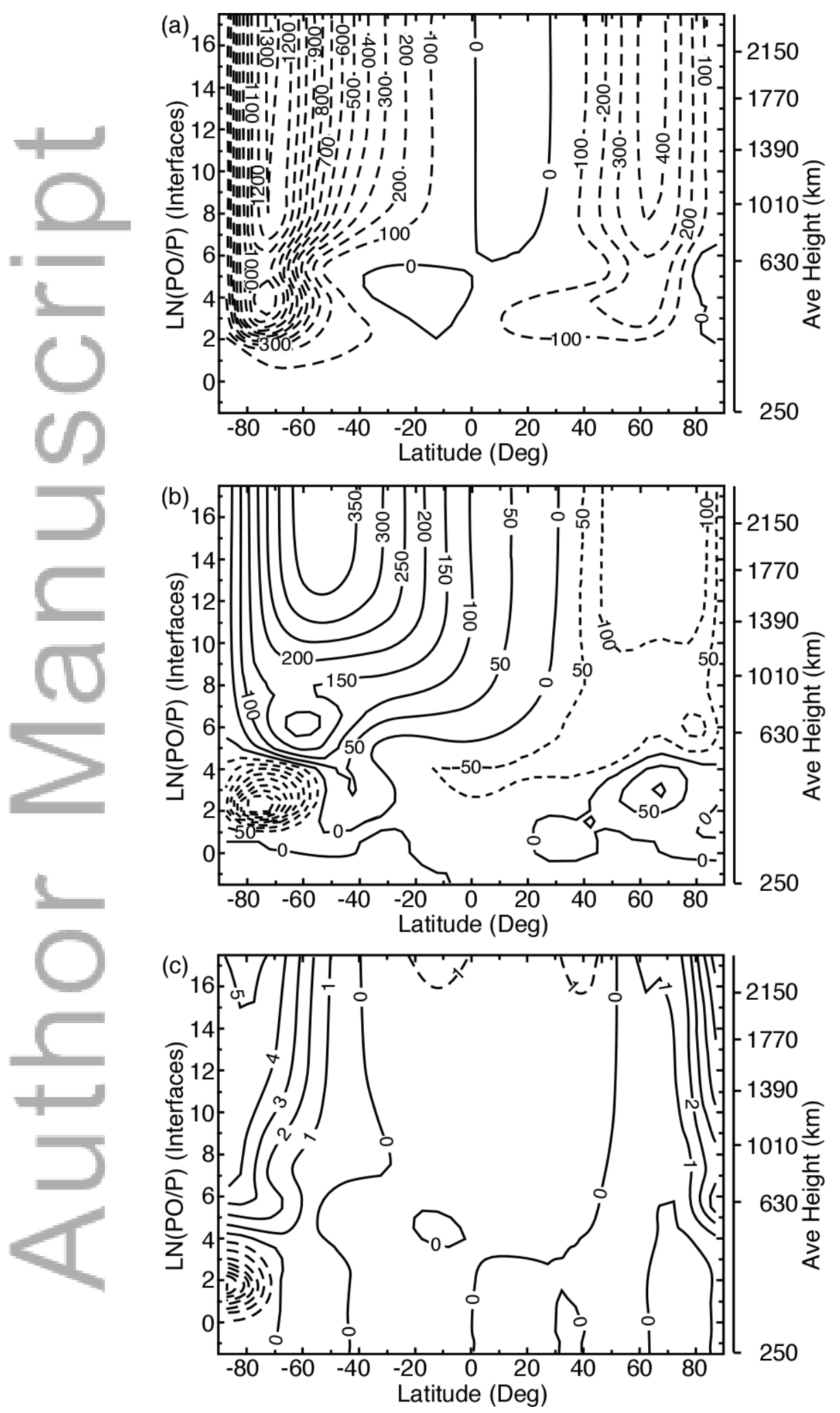

2015JA021328-f01-z-.tif 

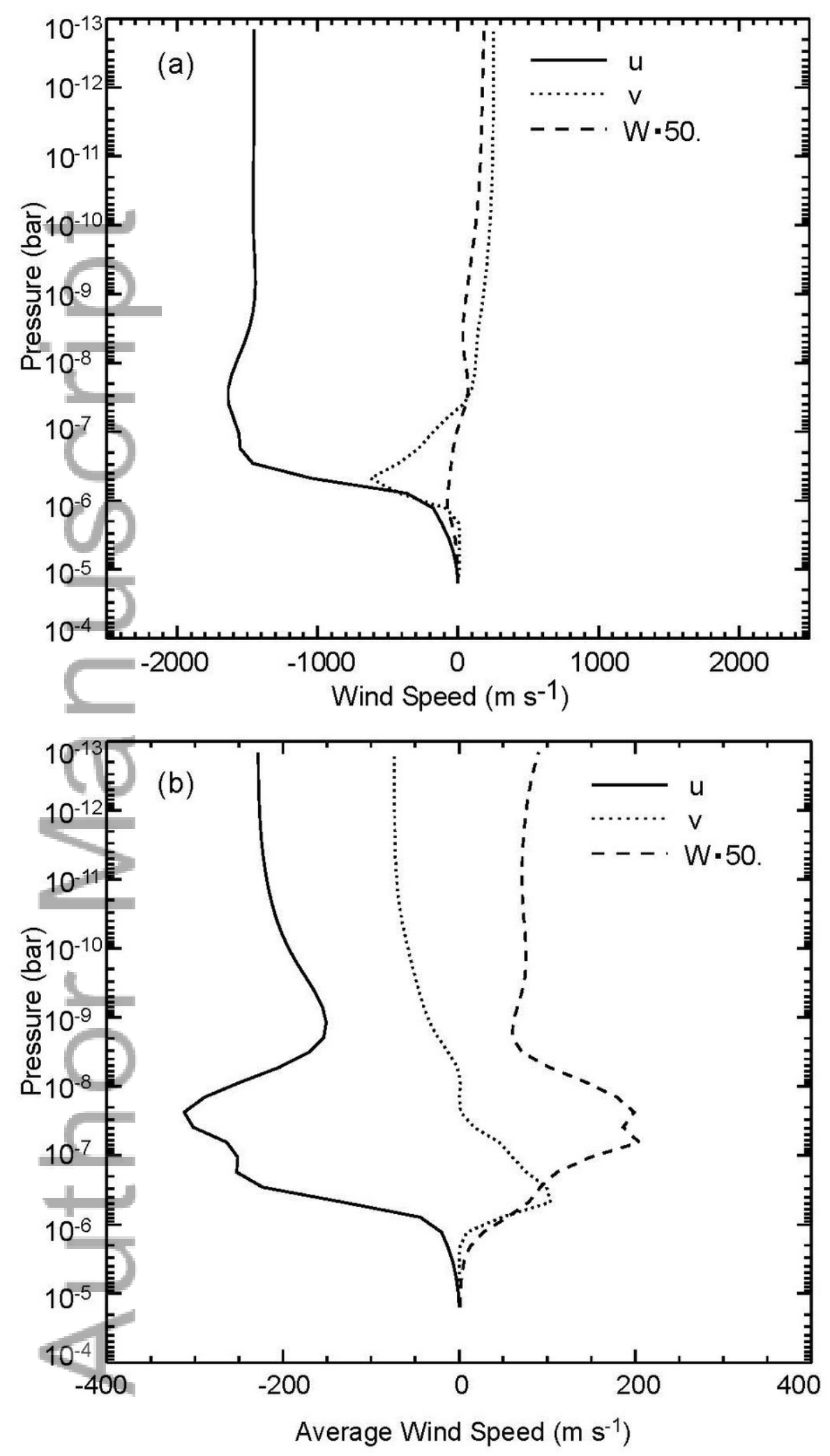

2015JA021328-f02-z-.jpg

This article is protected by copyright. All rights reserved. 


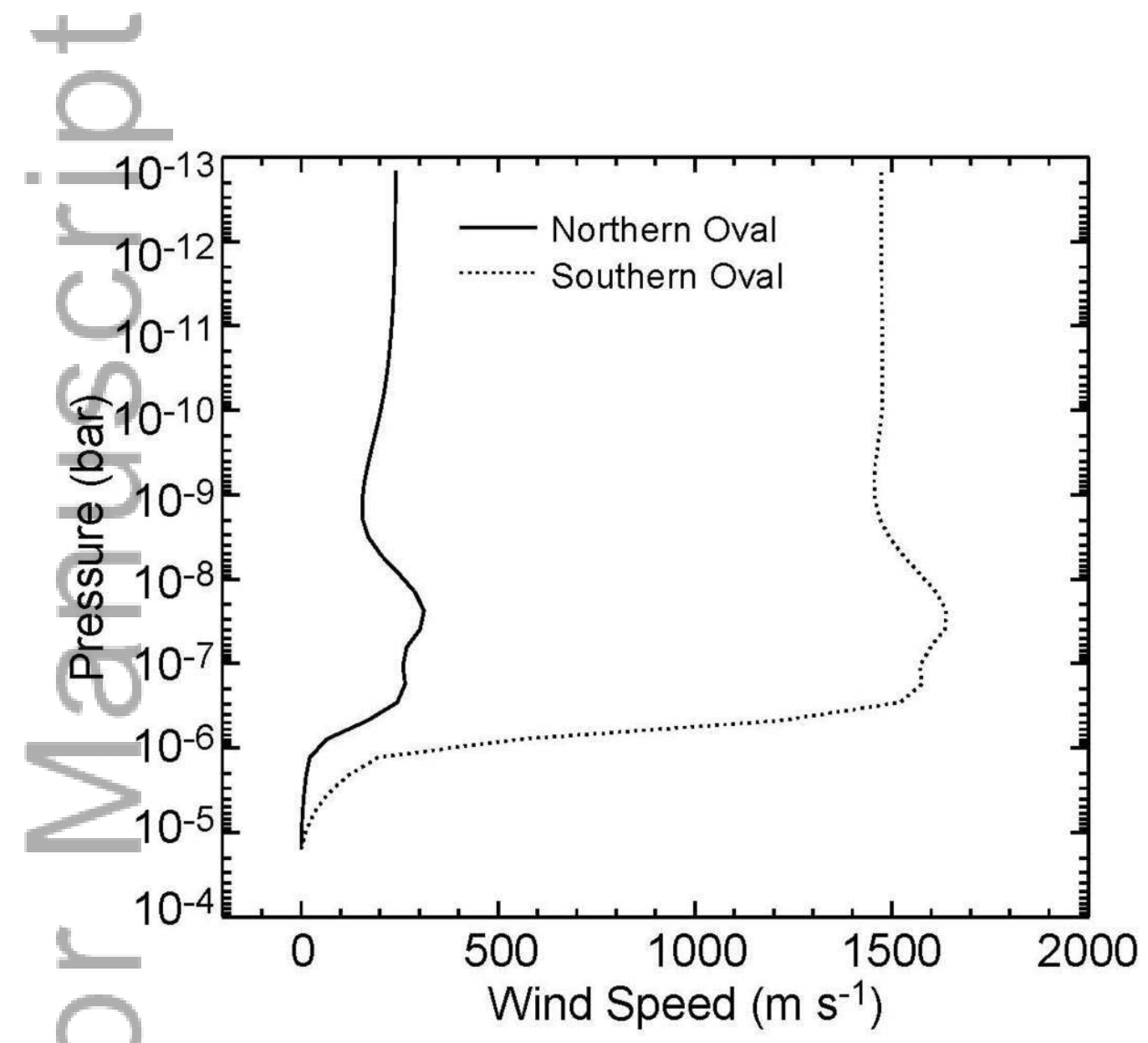

2015JA021328-f03-z-jpg

This article is protected by copyright. All rights reserved. 


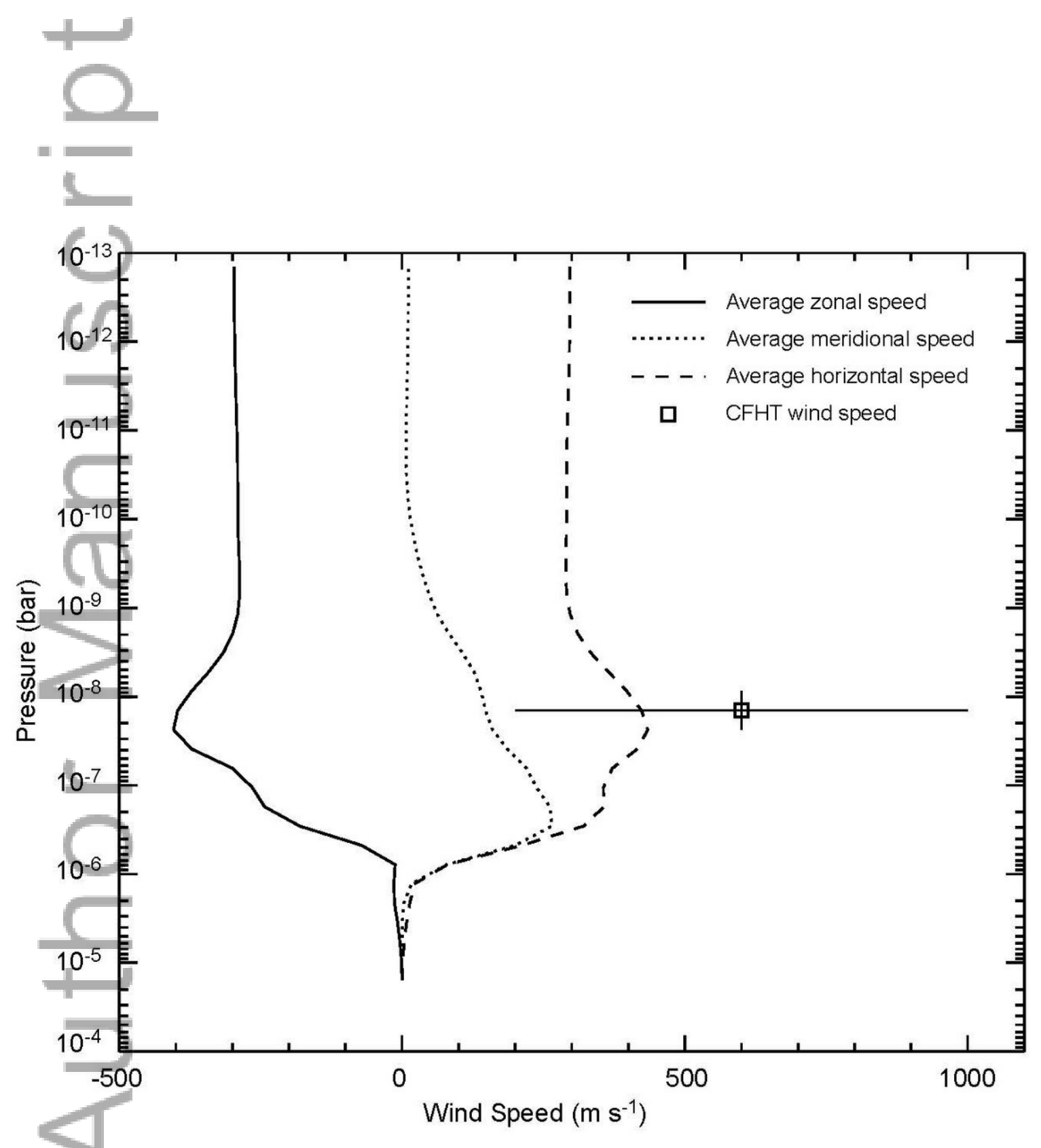

2015JA021328-f04-z-.jpg

This article is protected by copyright. All rights reserved. 

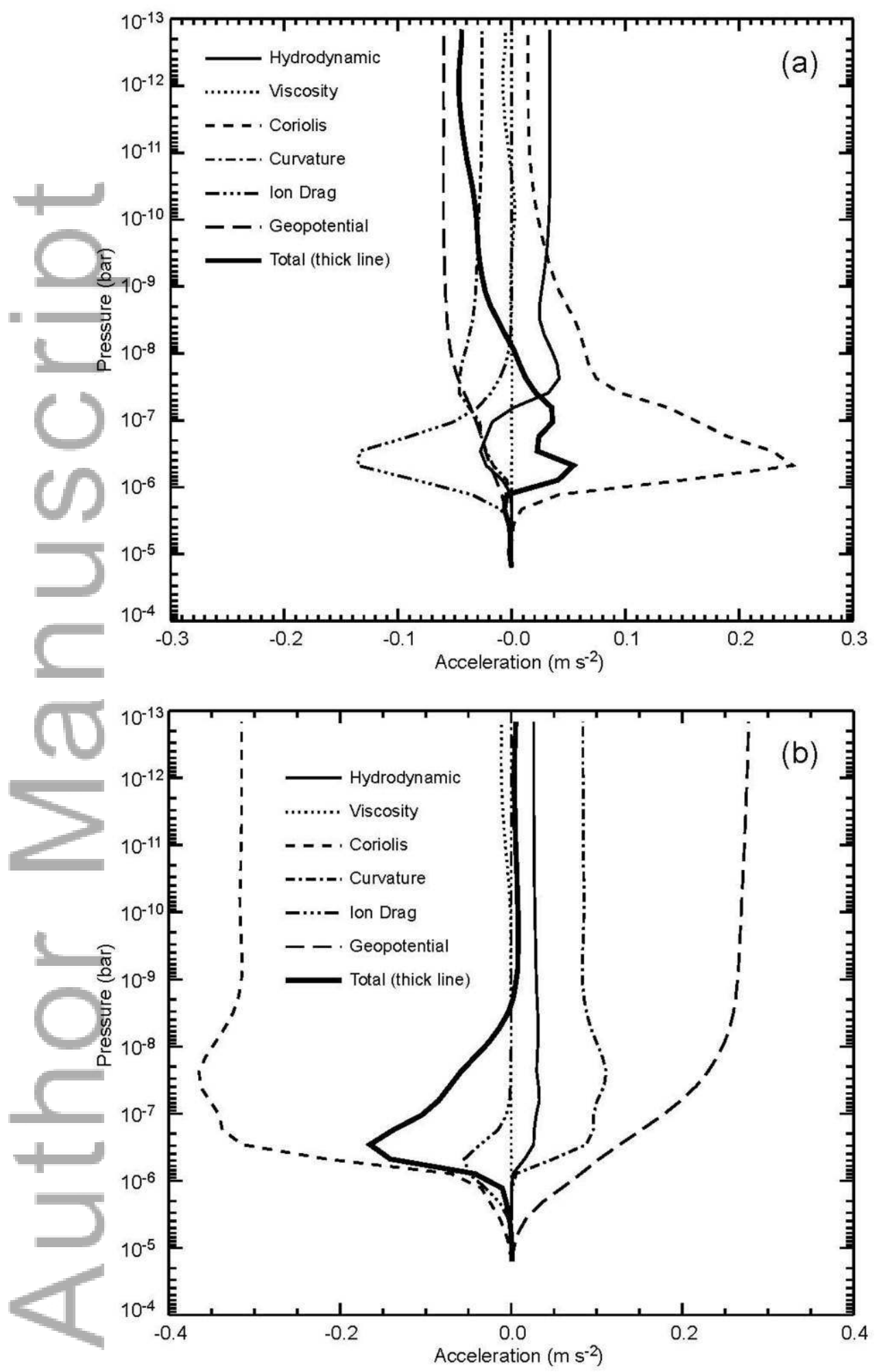

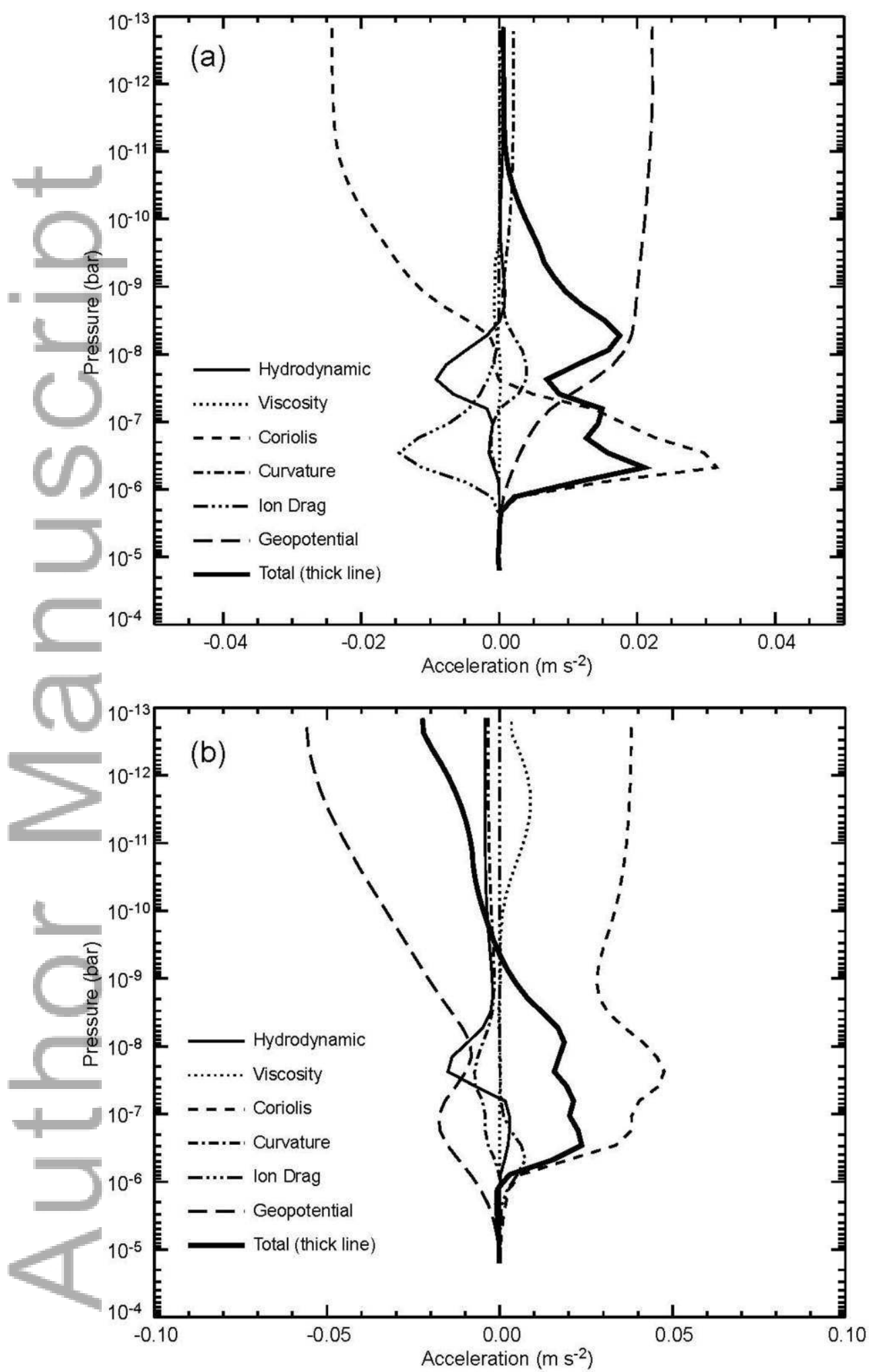

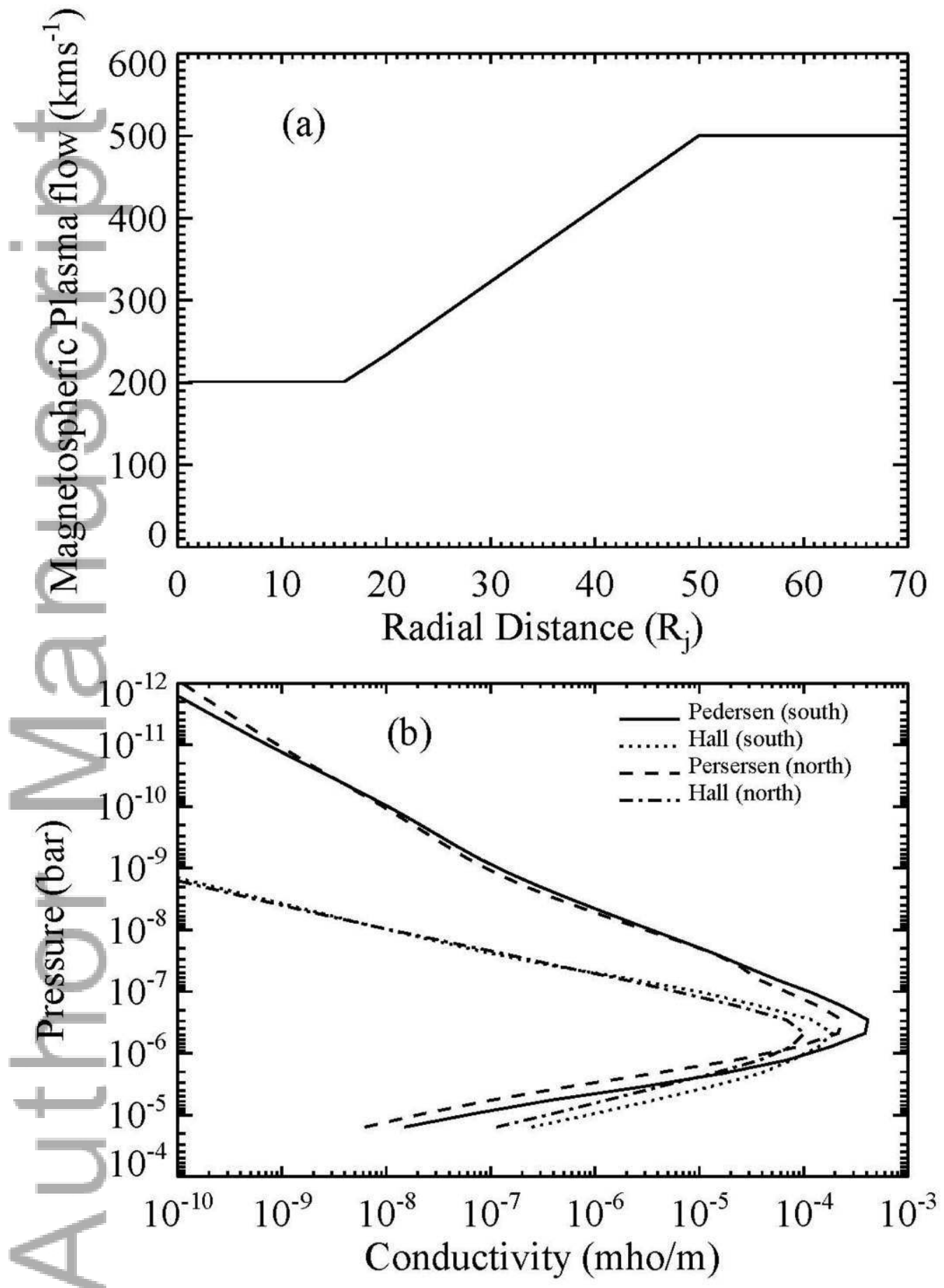

2015JA021328-f07-z-.jpg

This article is protected by copyright. All rights reserved. 